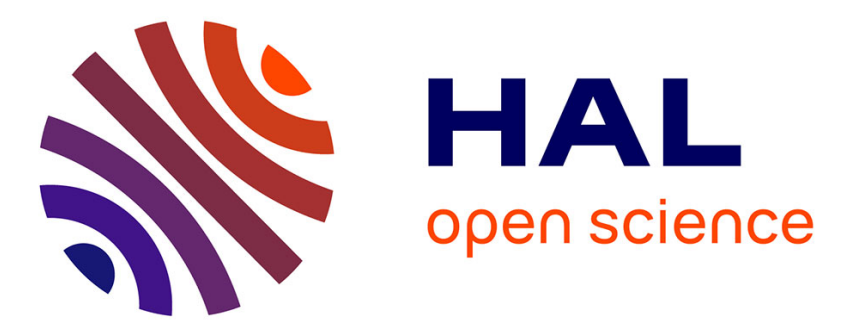

\title{
A reliable multipath routing protocol based on link quality and stability for MANETs in urban areas
}

\author{
S.E. Benatia, O. Smail, B. Meftah, M. Rebbah, Bernard Cousin
}

\section{To cite this version:}

S.E. Benatia, O. Smail, B. Meftah, M. Rebbah, Bernard Cousin. A reliable multipath routing protocol based on link quality and stability for MANETs in urban areas. Simulation Modelling Practice and Theory, 2021, 113, pp.102397. 10.1016/j.simpat.2021.102397 . hal-03337275

\section{HAL Id: hal-03337275 \\ https://hal.science/hal-03337275}

Submitted on 9 Sep 2021

HAL is a multi-disciplinary open access archive for the deposit and dissemination of scientific research documents, whether they are published or not. The documents may come from teaching and research institutions in France or abroad, or from public or private research centers.
L'archive ouverte pluridisciplinaire HAL, est destinée au dépôt et à la diffusion de documents scientifiques de niveau recherche, publiés ou non, émanant des établissements d'enseignement et de recherche français ou étrangers, des laboratoires publics ou privés. 


\title{
A Reliable Multipath Routing Protocol Based on Link Quality and Stability for MANETs in Urban Areas
}

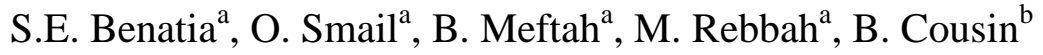 \\ ${ }^{a}$ Computer Science Department, Faculty of Exacte Sciences, University Mustapha Stambouli, Mascara, Algeria \\ \{se.benatia,o.smail,boudjelal.meftah,rebbahmed\}@univ-mascara.dz \\ ${ }^{b}$ IRISA, University of Rennes 1, Rennes, France \\ bcousin@irisa.fr
}

\begin{abstract}
A mobile ad hoc network (MANET) is a set of mobile and self-organizing nodes that cooperate to create dynamic network architecture to establish communications. Its characteristics present critical challenges: limited residual energy of nodes and transmission range, wireless links sensitivity to environmental effects, and the mobility aspect, which leads to frequent link failure and rapid changes in the network topology. In this paper, we propose a new multipath routing protocol RMQS-ua (Reliable Multipath Routing Protocol based on Link Quality and Stability in Urban Areas). Our objective is to select the path that has better link quality and more stable links to guarantee reliable data transmission. We consider a combination of signal to noise ratio SNR and an enhanced packet reception ratio PRR to evaluate link quality, and the exponential moving average (EMA) to estimate the link stability. RMQS-ua is designed for an urban area that includes shadowing effect and background noise which deteriorates the link quality. Simulation results show that RMQS-ua improves network performance, and provides more reliability compared to some recent existing protocols.
\end{abstract}

Keywords: Reliability, Multipath routing protocol, Link quality estimator, Link stability, Urban area, MANET

\section{Introduction}

Wireless communication technology has evolved rapidly in the last few decades due to the enhancement of its features: reliability and bandwidth have increased in order to meet the increasing demand. Wireless ad hoc networks are becoming an integral part of life, offering multiple advantages, such as the ability to be used anywhere and at any time independent of the device type (laptop, smartphone, etc.) [1].

A MANET (Mobile Ad hoc Network) is a specific wireless ad hoc network. It is composed of a set of mobile nodes having limited energy (generally powered by an electrochemical battery) that communicate with each other using wireless links, using a multi-hop strategy without the need of existing infrastructure or centralized control [2-4]. The nodes form a dynamic network that changes over time due to the mobility of the nodes and their limited transmission range.

The characteristics of ad hoc networks and the sensibilities of radio connectivity make communications between nodes a critical challenge; a source node may have to transmit data packets through intermediate nodes to reach the destination node. Hence, in order to ensure the reliability of data transmission, an efficient routing protocol must be implemented to find the best paths, taking into consideration several constraints such as link quality, delay, and throughput. Routing protocols are classified into three categories: proactive, reactive, and hybrid [1-3]. In a proactive protocol, the nodes maintain a global view of all nodes composing the network. The nodes periodically exchange topology messages, which contain data on nodes that are 1 hop and 2 hops away, to update their routing table. In contrast, a reactive protocol is an on-demand model based on a discovery and maintenance process using a requestresponse mechanism. It discovers routes by broadcasting a route request packet (RREQ) through the network to find a path between the source and the destination, and if a link breaks, the maintenance process is invoked. A hybrid protocol is a combination of both proactive and reactive protocols. A multipath technique has emerged; it is able to find multiple paths through the same discovery process, which may reduce the frequency of the discovery and maintenance processes.

Indeed, if several paths have been stored, when link breakage occurs on the active path, instead of triggering a new path discovery, the next best path among the stored paths is selected. Thus, the multipath technique may reduce reaction delay and packet overhead.

In the last few years, for the purpose of meeting different communication requirements, many routing protocols have been proposed based on several constraints. The basic constraint is minimizing the hop count. Several studies [1,5] 
proved the weakness of this metric; the minimum number of hops does not always reflect the optimal path since it ignores all other important factors (energy, link stability, signal quality, bandwidth, etc.) that have a considerable impact on packet transmission. In order to improve network performance, recent studies [2, 5-9] take into account specific metrics that influence data transmission, such as signal quality [5,10], bandwidth [11], mobility [7, 12-13], and energy $[6,14]$.

In a MANET, due to the unpredictable mobility of nodes, link breakage may occur frequently. These link breakages are not detected in real time, leading to packet losses. Consequently, the source node must broadcast discovery packets after each link breakage to find a new path. This overloads the network and exhausts the node batteries quickly. Therefore, inefficient routing decisions may significantly reduce the network's lifetime and performance. It is important to take this factor into consideration to select the optimal path.

Furthermore, another important factor that may lead to link failure is link quality. The radio links are affected by several factors that lead to the degradation of signal quality: noise (from the environment), interference, etc. [15]. The signal quality represents an active research area as the existing LQE (Link Quality Estimator) fails to provide sufficient accuracy. An LQE can be classified as physical-based or software-based; more details can be found in Section 2. The transmission range is defined by three regions around each node [15]: a connected region that contains stable links and has good link quality, a transitional region (called also intermediate region, it is the largest region) where links have intermediate quality and minor changes may affect the links, and a disconnected region.

In this paper, we propose a new reliable multipath routing protocol named RMQS-ua based on link quality and link stability metrics. RMQS-ua exploits alternative paths when the links break; this avoids relaunching a new paths discovery process costly in terms of delays, control messages, and energy consumption. The links quality evaluation considers the SNR (Signal Noise Ratio) which increase the accuracy of the quality estimator and gives us the ability to prevent link quality degradation, SNR is a physical based estimator who is combined with an enhanced PRR (Packet Reception Ratio) metric by taking into account successive packet losses rate, PRR is a software based estimator. We used a weighted quality estimator based on SNR and PRR in order to exploit the advantages of both physical and software metrics and avoid their weakness. SNR is a fast physical metric measured only for successfully received packets, PRR is a slow metric that requires previous data transmission and provides more efficiency.

In addition to link quality, the link stability is taken into consideration based on node mobility behavior to avoid link failure caused by mobility which increases the path lifetime. Our protocol is evaluated in an urban environment that has a restrictive mobility area. Several studies $[16,17]$ prove that a mobility model also has a considerable impact on the performance of routing protocols. The Manhattan Grid model is the most realistic model for an urban area because it simulates the mobility in a restricted area representing city streets. Another characteristic of an urban environment is that it affects the propagation of the radio signal (signal fading) and promotes noise. Finally, our solution provides better estimator accuracy path to evaluate the paths and selects the most stable path with better quality which decreases significantly the link failures, reduces packet loss and preserves the energy of nodes: thus, it improves network performance and ensures more data transmission reliability.

The paper is organized as follows. Section 2 presents a review of related works and provides an analysis of existing link quality estimators. In Section 3, we describe our routing protocol approach. Section 4 presents the performance evaluation; we evaluate our protocol compared to well-known routing protocols. Finally, Section 5 concludes this paper.

\section{Related works}

Ad hoc routing protocols must meet the main requirements of ad hoc networks, such as packet delivery rate, overhead, and end-to-end delay. Indeed, the researchers have focused on the most critical constraints that affect considerably network performance: link quality, stability, energy and load balancing.

In order to guarantee the communication quality and satisfactory network performance, routing protocols try to use an accurate metric that reflects the link quality. This metric is generally a numerical value based on several factors.

The estimation of wireless link quality to ensure reliable data transmission and satisfactory network performance is a major issue. LQE metrics can be classified into two categories: physical-based and software-based. Various routing protocols have been proposed in this context. 
Physical-based metrics are derived directly from the physical layer. Three physical LQEs exist: LQI, RSSI, and SNR.

- The RSSI (Received Signal Strength Indicator) defines the signal strength level of the received packet (in $\mathrm{dBm}$ ). Most radio interfaces provide a RSSI register [15].

- The SNR (Signal-to-Noise Ratio) defines the difference between signal power and the noise floor [18]. The SNR is better than the RSSI because it considers the noise floor.

- The LQI (Link Quality Indicator) provides information about signal quality; his is proposed in the IEEE 802.15 standard. The standard range of this metric is 0-255[19], but it may vary from 50 to 110 in specific cases [15].

Several models $[4,20]$ have been proposed based on physical metrics. The authors [4] have proposed a new model to predict the link quality using SNR sampling based on the grey method; this model is implemented on OLSR routing protocol. The protocol evaluation is carried out with nine static nodes. The obtained results show that the model improves some network performances. However, the PRR has a slight improvement compared to the other protocols. Also, they evaluate the protocol only on one specific case: nine static nodes where only the destination node moves. An enhancement of AODV and DSR has been proposed [2]; the proposed approach restricts the forward of control packets to only the nodes that satisfy a minimal threshold using two models: a zone model (based on RSSI) and segment model (based on link availability). The results show an improvement of the error packets number. Nevertheless, a very slight improvement is observed on overhead and bandwidth for AODV version. The authors do not discuss the Packet Delivery Rate or Packet Loss Ratio metrics and do not evaluate the model in a low-speed scenario.

Generally, the physical-based metrics do not require any extra computation or communication as they are extracted directly from the hardware device. They provide quick information about link quality, but this information is measured only by successfully received packet signals, which may overestimate the link quality. Moreover, physical metrics provide less accuracy due to their sensitivity, as the signal value may go up or down quickly due to sudden change. Some studies [2,21] prove that the correlation between the PDR (Packet Delivery Rate) and the RSSI decreases under some conditions, such as internal interference (interference results in low PDR values but increases the RSSI).

Software-based, or logical, metrics are obtained from the higher layers using packet transmission:

- The PRR (Packet Reception Ratio), similar to the PDR, is the ratio of successfully received packets over the number of packets sent [18].

- The RNP-based metric contains two metrics. The RNP (Required Number of Packets) counts the total number of packet transmissions or retransmissions before successful delivery of the packet [15]. It is better than the PRR because it takes packet retransmission into account. The ETX (Expected Transmission Count) estimates the number of retransmissions needed to send the packet by taking the asymmetry into consideration [1].

Several protocols [1,22] have been proposed based on the ETX metric. A modified version of the ETX named ETX_ANT (ETX anticipation) [1] has been developed: the authors use the RSSI to estimate the future FER (Frame Error Rate) and integrate it into ETX_ANT, which predicts the metric value in advance in order to improve the obtained result. However, the ETX_ANT is measured using broadcast messages; this may poorly estimate the link quality, as considerable control traffic is required in order to provide a good estimation. Thus, researchers [5] proposed a new variation of the ETX named the HETX (High load ETX), which measures the ETX of the previous time window instead of the last time window to overcome the impact of the flood(during discovery process) on the link quality estimation. The authors simulate different scenarios (varied number of nodes and different loads). The results show that HETX and EXT have approximatively the same results on normal and low loads and better performance on high load scenarios. However, this work is based only on static nodes which do not take into account the mobility aspect. Another variation of the ETX metric called the XDDR (estimated Directional Delivery Ratio) has been proposed [22]: it estimates the PRR over a fixed-length window using pulse quality selection and classification (using unicast instead of broadcast). It is implemented in both AODV and DSR routing protocols. The results show that XDDR version performs better than the other compared protocols.

Some researchers use other approaches to increase the network performance, such as machine learning [23], regression analysis [10], swarm optimization [24], and bio-inspired algorithms [9,25-26] which are integrated with multiple constraints to improve the accuracy of the quality measurement and the performance of the network. However, these methods generally require a long period of learning to provide better results, with more complex calculations. The 
authors [25] proposed a new multipath routing protocol by integrating the ant colony (ACO) algorithm in the selection process based on multiple constraints. They adapted the traditional ACO algorithm which uses only the pheromone as a factor by exploiting three metrics: link quality by RSSI, energy, and hop count. The proposed protocol improves network performance and extends network lifetime. However, it is compared only to the standard AODV protocol that uses hop count as a metric, where they should compare results other protocol that use multi constraints.

Other studies $[13,27]$ propose routing protocols based on the clustering method where the network is divided into clusters; each cluster is composed of a set of nodes that elect a node to be a cluster head $(\mathrm{CH})$. The cluster head takes control of operations inside the cluster. An energy efficient stable and secure clustering technique is presented [13]. The authors used a fuzzy logic model to select the cluster head based on five parameters: energy, degree (number of neighbors), distance, mobility, and trust level. It introduces a new concept named "Standby CH" which will be useful in case the cluster head moves out or dies. The simulation results show an important improvement in the number of cluster changes and overhead. However, the number of $\mathrm{CH}$ changes of the proposed protocol decreases and present a slight improvement when the node speed increase. Also, it is not evaluated under different network densities (only the case of 100 nodes is used).

Furthermore, link stability and energy constraints are the subjects of several works. Link stability is generally measured using a metric or method that takes node mobility into account. A new multipath energy-efficient routing protocol using a Fitness Function (FF-AOMDV) [6] has been proposed. This protocol is based on the standard AOMDV protocol and integrates both energy and mobility constraints and hop count. The proposed approach selects the path having a high energy level and the minimum distance to the destination which reduces the energy consumption and endto-end delay. However, the high energy level path may be composed of an energy exhausted node, which leads to node failure. Moreover, this approach does not take the path stability and the link quality into account, so the selected path may be broken quickly. The authors [28] proposed a new routing protocol, based on link stability and energy awareness, named LSEA. The main idea is to ensure that nodes have enough residual energy and link lifetime before forwarding the request packet the discovery process. Two versions have been proposed: A-LSEA (Average-LSEA) based on the average of all neighbors as a threshold and F-SLEA (Fixed-LSEA) based on a defined threshold. The simulation results show that A-LSEA outperforms F-LSEA and the standard AODV (evaluated using only 100 nodes). Nevertheless, even when a stable path (in mobility matter) with sufficient energy is selected, the communication may be ineffective with high packet loss, because these techniques ignore the radio signal quality, which can be affected by the environment.

Due to the limitations of using one metric (software or hardware), the researchers are focused on using combinations of multiple metrics in order to improve QoS and network performance by the selection of the optimal path. Many works [11,29-31] has been presented in this context. DTRP protocol [29] (Decision Tree-based Routing Protocol ) has been proposed. The contribution is on the route construction during the flooding request using a decision tree calculated based on multiple parameters (node lifetime, speed ...). However, the path is not evaluated when we have multiple paths, and they did not consider any link quality factor related to wireless signal. The authors in [30] proposed a new routing protocol named TA-AOMDV, which is based on multiple constraints (link stability, bandwidth,..). The path selection process chooses the most stable path based on PSP (path stability probability). The simulation results show that the proposed protocol is better than the other protocols. However, it generates a high overhead.

The authors in [32] analyze the availability and the duration probability of paths that may be subject to link breakage due to the mobility of the nodes. They propose a mathematical model in order to improve path availability according to the Random Direction mobility model. However, this requires some assumptions (i.e., the received signal is dependent only on the distance from the transmitter) that are unrealistic; its specification for only one mobility model is a limitation of this approach. Others studies have been proposed based on energy constraints [14] and link stability [33], disregarding the impact of wireless quality.

In addition to the drawbacks cited for each protocol, most literature routing protocols have been evaluated using random mobility models mainly Random Waypoint model (RWP), this model considers unrealistic behaviors such as sudden stops and abrupt accelerations. In a restricted urban environment affecting the radio signal propagation and generating noise, the protocol solutions presented are unsuitable for such environments and may produce poor performance. 


\section{Reliable Multipath Routing Protocol Based on Link Quality and Stability in Urban Areas}

We describe in this section a new multipath routing protocol named RMQS-ua, which takes into account link quality and link stability constraints. These constraints are combined in a metric used to classify the discovered paths and select the path that ensures the most reliable data transmission. In order to avoid generating additional overhead, we have exploited the control packets of the routing protocol and adapted its structure to collect some required information (like the distance between two nodes); this information will be used to evaluate links composing a path.

The selection of a stable, high quality path helps us to maximize the network performance and maximize the lifetime of the selected path, which decreases the number of link breakages and packet losses. Therefore, we reduce the huge resource consumption produced by the repeated discovery process in terms of energy, bandwidth, overhead, and so on.

\subsection{Link quality estimator discussion}

We describe briefly in Section 2 the existing Link Quality Estimators (LQEs) and their classes, and we notice that each class has different advantages and limitations. The physical-based metrics generally provide a quick estimator, as they measure directly from the hardware device with the need for only minimal computation. However, they are less accurate; especially in the intermediate (transitional) region as their value may changes suddenly from high to low. Additionally, link quality is measured only on the successfully received packets, and that may overestimate the link quality by ignoring the lost packets. Several previous works prove that the SNR provides a better metric than the RSSI because it takes into consideration not just the signal strength, but also the background noise. For that reason, we decided to use the SNR metric.

In contrast to physical metrics that can be measured on each received signal, the software-based metrics require a set of received packets to evaluate the link quality. This means that their computations are slow, but they provide a more accurate average measurement. Therefore, this type of metric is not fit to produce the first routing decision because it requires previous traffic to be sent in order to estimate the link quality. Otherwise, in both cases, it is difficult to measure the link quality if the link is not used.

We conclude that to propose a reliable quality estimator, we have to combine both a hardware metric (we choose the SNR) and a software metric (we choose the enhanced PRR) using a dynamic weighting factor to estimate the link quality. We also used link stability metric as a second metric based on EMA (Exponential Moving Average). The link quality and link stability metrics help us decrease the link failure rate, which increases the path lifetime, improves the data delivery rate, and reduces the overhead and energy consumption.

\subsection{Problem Formulation}

A MANET is a set of nodes connected to each other using wireless links. This network can be represented as an undirected graph $G=(N, L)$, where $N=\left\{n_{0}, n_{1}, \ldots.\right\}$ is a set of nodes composing the network, and $L=\left\{l_{0,1}, l_{l, i}, \ldots \ldots\right\}$ is the set of links (with $l_{i, j}$ defines the link composed by $n_{i}$ and $\left.n_{j}\right)$. Let $P_{k}\left(n_{0}, n_{m}\right)=n_{0}, n_{k l}, \ldots n_{m}$ be the $\mathrm{k}^{\text {th }}$ path in the set of paths $P\left(n_{0}, n_{m}\right)$, where the source node is denoted by $n_{0}$ or $n_{k 0}$ and the destination node is denoted by $n_{m}$ or $n_{m 0}$.

Regarding the mobility of nodes, we use Euclidian distance. Thus, the distance between two nodes $n_{i}$ at coordinates $\left(x_{i}, y_{i}\right)$, and $n_{j}$ at coordinates $\left(x_{i}, y_{i}\right)$ at time $t$ is defined as the following:

$$
d_{i, j}(t)=\sqrt{\left(x_{i}(t)-x_{j}(t)\right)^{2}+\left(y_{i}(t)-y_{j}(t)\right)^{2}}
$$

In wireless network, the propagation of the radio signal can be affected by different factors (such as noise) which degrade its quality. The transmitted packet is characterized principally by its signal strength.

Let $n_{i}$ represents the upstream node and $n_{j}$ represent the downstream node. For each link $l_{i, j} \in L$, the SNR (Signal-toNoise Ratio) at time $t$, according to the most widely used radio propagation model $[4,34]$ can be measured for each received packet as following:

$$
S N R_{i, j}(t)=P_{t r}-P L\left(d_{0}\right)-10 * n * \log \left(\frac{d_{i, j}(t)}{d_{0}}\right)-X_{\sigma}-P_{n}(2)
$$

where $P_{t r}$ represents the transmission power of the received packet, $P L\left(d_{0}\right)$ is the path-loss at the reference distance $d_{0}$ (usually equal to $1 \mathrm{~m}$ ), $X_{\sigma}$ is a Gaussian random variable with mean $=0$ (representing shadow fading). 
Based on [4] and [34], the $\operatorname{RSSI}_{i, j}(t)$ (Reception Signal Strength Indicator) for each link $l_{i, j}$ at time $t$ is given by:

$$
\operatorname{RSSI}_{i, j}(t)=P_{t r}-P L\left(d_{0}\right)-10 * n * \log \left(\frac{d_{i, j}(t)}{d_{0}}\right)-X_{\sigma}(3)
$$

Let $P_{n}$ represent the background noise (in $\mathrm{dBm}$ ) of the link $l_{i, j}$ at time $t$. Then, from the equations (2) and (3): for each received packet, the $S N R$ can be expressed as follow:

$$
\operatorname{SNR}_{i, j}(t)=\operatorname{RSSI}_{i, j}(t)-\text { Noise }_{i, j}(t)
$$

The PRR (Packet Reception Ratio) at time $t$ of the link $l_{i, j}$ is given by:

$$
\operatorname{PRR}_{i, j}(t)=\frac{N R_{i, j}(t)}{N S_{i, j}(t)}
$$

Where $N R_{i, j}(t)$ defines the number of packets received successfully by $n_{j}$ from $n_{i}$ till time $t$, and $N S_{i, j}(t)$ defines the number of packets transmitted from $n_{i}$ to $n_{j}$ till time $t$.

\subsection{Multipath Discovery Process}

The route discovery process for the on-demand multipath routing protocol has the objective to find multiple paths to establish a communication between a source and a destination node. The RMQS-ua protocol uses the same control messages as AOMDV [35]: a RREQ (Route Request message) and a RREP (Route Reply message) are used in the AOMDV discovery process, and RERRs (Route Error messages) and Hello messages are used in the AOMDV maintenance process.

However, in order to adapt the control messages used in the AOMDV protocol to our approach according to the link quality and link stability factor computations, additional fields have been added to these messages. For the Hello message, $C_{j}(t)$ represents the coordinates of the node $n_{j}$, the neighbor of the intermediate node $n_{i}$ at time $t$. For the RREQ and RREP packets, $f s(t)$ and $f q(t)$ have been added, where $f s(t)$ represents the maximum stability value of links composing a path, and $f q(t)$ represents the minimum quality value. For all messages, the source and destination fields designate the source node and the destination node, respectively, and the sequence number field is used to ensure the freshness of the paths (detailed below). Fig. 1 and Fig. 2 present, respectively, the structures of the Hello message and the RREQ message of our protocol.

\begin{tabular}{|c|l|c|c|}
\hline Destination & $\begin{array}{l}\text { Destination } \\
\text { Sequence number }\end{array}$ & $C_{j}(t)$ & $\begin{array}{c}\text { Expiration } \\
\text { timeout }\end{array}$ \\
\hline
\end{tabular}

Fig. 1. Structure of Hello message of RMQS-ua protocol

\begin{tabular}{|l|l|l|l|l|l|l|}
\hline REQ_ID & $\begin{array}{l}\text { Destination } \\
\text { Sequence } \\
\text { number }\end{array}$ & Destination & $\begin{array}{l}\text { Source } \\
\text { Sequence } \\
\text { number }\end{array}$ & Source & $f s_{i, j}(t)$ & $f q_{i, j}(t)$ \\
\hline
\end{tabular}

Fig. 2. Structure of RREQ message of RMQS-ua protocol

In addition to the control messages, the routing table is also changed (both stability function $f s(t)$ and quality function $f q(t)$ values are added) as shown in Fig. 3.

\begin{tabular}{|l|}
\hline Destination \\
\hline Destination sequence number \\
\hline Advertised_hopcount \\
\hline Route_list \{ \\
(Nexthop1,Hop-count 1, $f s(t), f q(t)) ;$ \\
(Nexthop2,Hop-count2, $f s(t), f q(t)) ; \ldots \ldots\}$ \\
\hline Expiration timeout \\
\hline
\end{tabular}

Fig. 3 Structure of routing table entry of RMQS-ua protocol of node $i$ 
When a source node $S$ intends to send a data packet to a specific destination node $D$, and it does not have any available path toward this destination in its routing table, then $S$ launches a path discovery by broadcasting a Route Request message (RREQ) to all its one-hop neighbors. It also sets a timer RREQ_Timer to detect the case where $D$ is inaccessible.

When an intermediate node receives a RREQ message, it ensures that the received message is not duplicated by examining the RREQ identifier REQ_ID: thus, only a unique RREQ (the first received RREQ) will be forwarded by the intermediate node. After that, the intermediate node compares the $f s$ and $f q$ values from RREQ with the stored values (in the routing table) of stability and quality, respectively, of the link connecting this node with the preceding node (which forwarded the RREQ), updates $f q$ by the minimum value (the poorest quality) and $f s$ by the maximum value (the worst stability), and then maintains a reverse path to the source node. If the intermediate node has a valid path toward the destination, it generates a Route Reply message (RREP) and sends it back through the reverse path toward the source node. Otherwise, it increments the hop-count field and forwards the RREQ to its neighbors. The same process will be repeated for each intermediate node until we reach the destination node, or when the RREQ_Timer expires without receiving any REEP.

Once the destination node gets a RREQ message, it creates the reverse path to the source node, and then sends a RREP message with the minimum value of $f q$ and the maximum value of $f s$ toward to the source $S$. The destination $D$ responds for each RREQ message received by generating a RREP message and sends it to the source node. Algorithm 1 describes this process.

Algorithm 1 RMQS-ua: $j$ receives a RREQ from $i$ for destination $d$

Let $s$ : source node, $d$ : destination node, $i$ : the forwarding node.

if $\left(\mathrm{RREQ}[\mathrm{SeqN}]>\mathrm{SeqN}^{\mathrm{s}}\right)$ then // check if is a freshest route

Remove all reverse paths to $s$ from routing table

$\mathrm{SeqN}^{\mathrm{s}}:=\mathrm{RREQ}[\mathrm{SeqN}]$

advertised_hop ${ }^{\mathrm{s}}=$ infinite

Create a reverse path to $s$ through $i$ with (RREQ [hop-count] +1)

else if $\left(\mathrm{RREQ}[\mathrm{SeqN}]=\mathrm{SeqN}^{\mathrm{s}}\right)$ then

Insert a new reverse path to $s$ through $i$ with (RREQ [hop-count] +1)

else Drop RREQ endif

if $((j=d)$ or $(j$ have a valid route to $d))$ then

// if is the destination node or know a route to destination

generate RREP having

$\operatorname{RREP}[\mathrm{fs}]=\max \left(\operatorname{RREQ}[\mathrm{fs}], \mathrm{fs}_{\mathrm{i}, \mathrm{j}}(\mathrm{t})\right) \quad$ and $\quad \mathrm{RREP}[\mathrm{fq}]=\min \left(\mathrm{RREQ}[\mathrm{fq}], \mathrm{fq}_{\mathrm{i}, \mathrm{j}}(\mathrm{t})\right)$

Send RREP to $s$ through $i$

else if (is not duplicated RREQ) then

// if is first REQ_ID received

RREQ[hop-count] ++
RREQ[fs] $=\max \left(\operatorname{RREQ}[f s], \mathrm{fs}_{\mathrm{i}, \mathrm{j}}(\mathrm{t})\right)$
$\mathrm{RREQ}[\mathrm{fq}]=\min \left(\mathrm{RREQ}[\mathrm{fq}], \mathrm{fq}_{\mathrm{i}, \mathrm{j}}(\mathrm{t})\right)$
Re-broadcast RREQ to all neighbors

else drop RREQ endif

// if REQ_ID is already received before

Furthermore, at each node, the source or destination node sequence number $(\operatorname{SeqN})$ should be verified in order to guarantee the freshness of path data and avoid a path loop. If the control message received by an intermediate node has a sequence number with a higher value than the value stored in the routing table for the same path, then it removes all existing reverse paths and creates a new path entry.

The RREP gets back to an intermediate node through the reverse path to the source node. For our protocol, as it may find multiple paths, when the source node receives the first RREP message, it sets a timer RREP_Timer and waits a specific time to collect other RREPs before it starts sending data, as is described in Algorithm 2. 
Algorithm 2 RMQS-ua: $j$ receives a RREP from $i$ for destination $d$

\section{if $\left(\mathrm{RREP}[\mathrm{SeqN}]>\mathrm{SeqN}^{\mathrm{d}}\right)$ then}

// if is a freshest route

Remove all reverse paths to $d$ from routing table

$\mathrm{SeqN}^{\mathrm{d}}=\mathrm{RREP}[\mathrm{SeqN}]$

advertised_hop $^{\mathrm{d}}=$ infinite

Create a reverse path to $d$ through $i$ with (RREP [hop-count] +1)

else if $\left(\mathrm{RREP}[\mathrm{SeqN}]=\mathrm{SeqN}^{\mathrm{d}}\right)$ then

Insert a new reverse path to $d$ through $i$ with (RREP [hop-count] +1)

endif

if $(j=s)$ then

// if is the source node

if (is the first RREP) then set RREP_Time

// wait to collect multiple RREP if exist

if (RREP_Time is expired) then start sending data packet through the optimal path

else

RREP[hop-count] ++

Forward RREP to next hop

endif

At the expiration of the timer RREP_Timer, the source node evaluates the different available paths based on the route metric value, selects the best path (that have the higher objective function value) according to the proposed objective function (more details in the next section), and then starts data transmission through it.

When the broadcast timer RREQ_Timer expires and the source $S$ has not received any RREP message, it starts a new discovery process and broadcasts a RREQ message with an incremented destination_sequence_number value (SeqN++) to the whole network again. MAX_RREQ_Retries defines the maximum number of discovery tries allowed. If we reach the maximum number of tries without receiving any RREP message, then the destination node will be considered as an unreached node.

\subsection{Multipath selection}

Once the RREP_Timer expires, the source node must choose one path to transmit the data packets. However, because of the mobility of the nodes and the possible degradation of the link quality over time, one or several path links may be broken or unstable. In our approach, we classify the paths according to link quality and link stability in order to improve the performance of the routing protocol.

\subsubsection{Link quality function}

As we have discussed in Section 3.1, the quality function, defined as $f q_{i, j}(t)$, is a combination of two link quality estimators: the SNR (physical-based) and the PRR (software-based).

In order to give a good estimation of the PRR, we take the successive packet loss into consideration as a factor that affects the link quality. Links may be unstable due to external environmental factors, node mobility, or radio frequency interference. For these reasons, successive packet loss is used to estimate link quality.

$P R R \_e n h_{i, j}(t)$ denotes the packet reception ratio received by node $n_{j}$ from node $n_{i}$ :

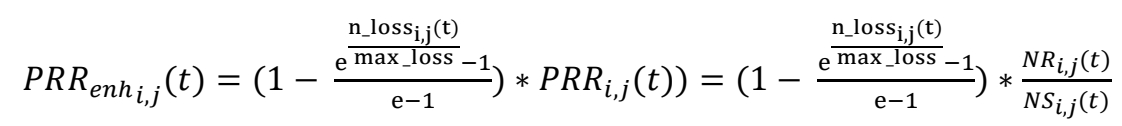

Where $n \_l o s s(t)$ is the number of successive packets lost, and max_loss is the maximum number of packets allowed to be lost to consider the link unreliable. 
We have chosen an exponential weight based on successive packet loss as $e^{\frac{n_{-l o s s}, j(t)}{\text { max } \_l o s s}}$. This increases the weight exponentially if multiple successive packets have been lost, but we know that $1 \leq e^{\frac{n_{-} \text {loss }_{i, j}(t)}{\text { max } \_l o s s_{-}}} \leq e$.

In order to ensure that the weight range is between 0 and 1, we use the max-min normalization (called also upperlower bound normalization) giving by:

$$
g_{n}(x)=\frac{g(x)-g_{\min }(x)}{g_{\max }(x)-g_{\min }(x)}
$$

If we take: $g_{i j}(t)=e^{\frac{n_{-} \operatorname{loss}_{i, j}(t)}{\text { max }_{-} \text {loss }}}$; we obtain:

$$
g_{i j}(t)=\frac{e^{\frac{n_{-} \text {loss }_{i, j}(t)}{\text { max } l o s s}}-1}{e-1} \quad ; \text { As } \begin{cases}\text { if } \mathrm{n}_{-} \operatorname{loss}_{\mathrm{i}, \mathrm{j}}(\mathrm{t})=0 & \text { then } g_{\min }(t)=1 \\ \text { if } \mathrm{n}_{-} \operatorname{loss}_{\mathrm{i}, \mathrm{j}}(\mathrm{t})=\text { max_loss } & \text { then } g_{\max }(t)=e\end{cases}
$$

Thus, in order to have a decreasing weight when the number of successively lost packets increases, we used $\left(1-g_{i j}(t)\right)$ instead of $g_{i j}(t)$. The exponential weight is formulated so that when $n \_l o s s(t)$ tends to 0 , then the weight tends to 1 , and when $n \_l o s s(t)$ tends to max_loss, then the weight tends to 0 . Thus:

$$
1-g_{i j}(t)=1-\frac{e^{\frac{n_{\text {_loss }}, j(t)}{\text { max }_{-} \text {loss }}}-1}{e-1}
$$

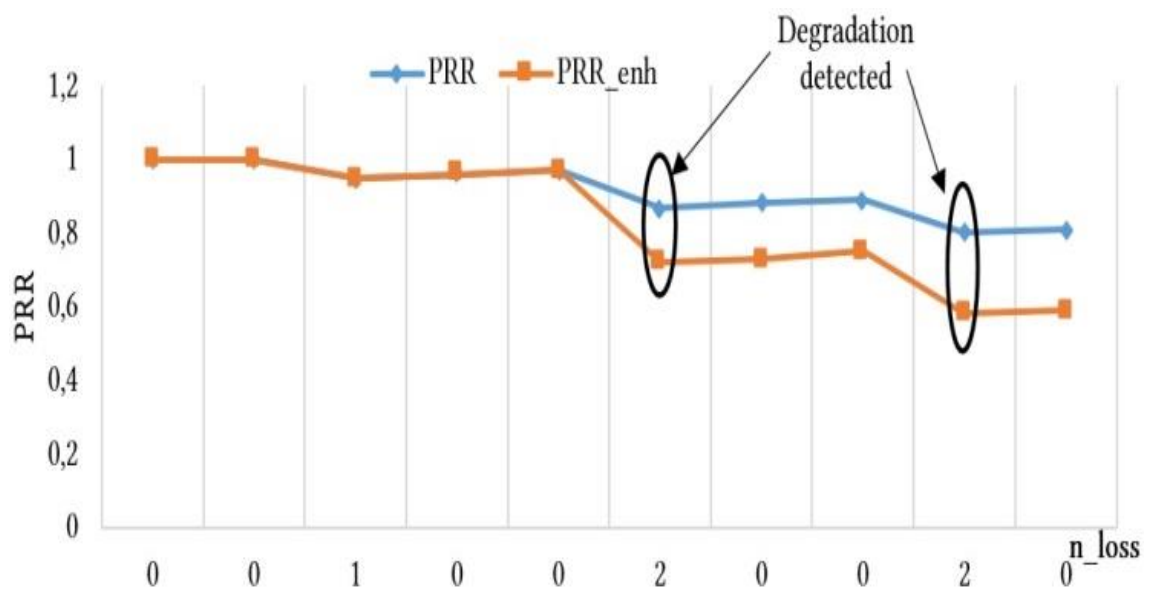

Fig. 4. Comparison between PRR and PRR_enh

Fig. 4 shows the difference between the standard PRR and PRR_enh (our solution). The loss of multiple successive packets indicates that the link is being unstable, which may produce an increased probability of link breakage. The results show that the value of PRR_enh decreases significantly in this situation in order to detect the link state changes rapidly.

We design a dynamic weighted factor $\alpha$ for the quality function $f q_{i, j}(t)$. The SNR can be used for a fast estimator for link quality. To exploit the reliability of the $P R R$, which requires the presence of a transmitted packet, the weight of the factor $\alpha$ is increased exponentially. For the initial state, where the number of sent packets is null or very low, $\alpha$ gives the full weight to the SNR. Then, it decreases over the time, preferring the $P R R$ (the $P R R$ provides more accurate information when the number of transmitted packets is important) as the number of packets transmitted increases.

For a given link $l_{i, j}$, the size of the packet window is denoted by $w$. The link quality function $f q_{i, j}(t)$ is given by:

$$
f q_{i, j}(\mathrm{t})=\alpha * P R_{e^{2 n h}}(\mathrm{t})+(1-\alpha) * \frac{\operatorname{SNR}_{i, j}(t)}{\operatorname{Max}_{S N R} R_{i, j}} \quad \text { where } \alpha=q *\left(2^{\frac{n b r_{-} p}{w}}-1\right),
$$

Where $q$ is a threshold of the $\alpha$ factor, $n b r \_p$ is the number of packets received in a packet window $w$, and Max_SNR is the maximum observed value of the SNR. 


\subsubsection{Link stability function}

In order to estimate the link stability, we consider the mobility of the nodes. A given node $n_{i}$ periodically broadcasts a Hello message to all its neighbors that contains its coordinates. Once a neighbor $n_{j}$ receives the Hello message, it calculates the Euclidian distance $d_{i, j}(t)$ between itself and node $n_{i}$ and then updates the stability cost $f s_{i, j}(t)$.

Let two nodes be denoted as $n_{i}$ and $n_{j}$. $E M A_{i, j}(t)$ represents the exponential weighted moving average of successive distances between $n_{i}$ and $n_{j}$ at time $t$ and is given by:

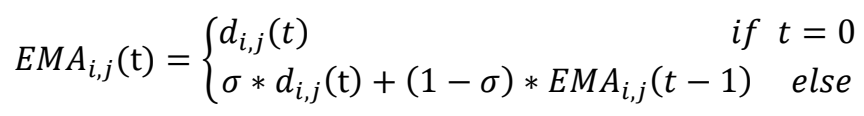

where the range of the weight coefficient $\sigma$ is between 0 and 1 .

We compute $\delta=d_{i, j}(t)-E M A_{i, j}(\mathrm{t}-1)$

$$
\operatorname{Var}_{i, j}(\mathrm{t})=\left\{\begin{array}{lr}
0 & \text { if } t=0 \\
(1-\sigma) *\left(\operatorname{Var}_{i, j}(\mathrm{t}-1)+\sigma * \delta^{2}\right) & \text { else }
\end{array}\right.
$$

The advantage of using the variance based on EMA is that it can be computed quickly based on previous data samples while preserving the node storage, as it requires only the last observed sample to be saved.

$f s_{i, j}(t)$ is based on the relative standard deviation. It denotes the link stability function between node $n_{i}$ and node $n_{j}$ at $t$ :

$$
f s_{i, j}(t)=\frac{\sqrt{\operatorname{Var}_{i, j}(t)}}{R-E M A_{i, j}(t)}
$$

Where $R$ represents the maximum transmission range of nodes; the stability function measures the dispersion of a distribution series. When the distribution tends to zero, this trend indicates a good distribution of distances, showing that the link is stable.

\subsubsection{The objective function}

The objective function combines the link quality and link stability functions to evaluate the available paths and determine the most reliable path.

For a given path $P_{k\left(\mathrm{n}_{0}, \mathrm{n}_{\mathrm{m}}\right)}$ between the source node $n_{0}$ and the destination node $n_{m}$, the objective function $f_{k\left(n_{0}, n_{m}\right)}$ is given by:

$$
\begin{aligned}
f_{k\left(n_{0}, n_{m}\right)}=100 * \beta * f q p_{k\left(n_{0}, n_{m}\right)}+\frac{(1-\beta)}{f s p_{k\left(n_{0}, n_{m}\right)}} \text { with } \beta \in[0,1] \\
\text { With }\left\{\begin{array}{l}
f q p_{k\left(\mathrm{n}_{0}, \mathrm{n}_{\mathrm{m}}\right)}=\min _{\mathrm{n}_{0}}^{\mathrm{n}_{m}}\left\{f q_{i, j}(t)\right\} \\
f s p_{k\left(\mathrm{n}_{0}, \mathrm{n}_{\mathrm{m}}\right)}=\max _{\mathrm{n}_{0}}^{\mathrm{n}_{\mathrm{m}}}\left\{f s_{i, j}(\mathrm{t})\right\}
\end{array} \text { where } l_{i, j} \in p_{k} .\right.
\end{aligned}
$$

The coefficient $\beta$ depends on the quality function. We define $\beta$ as the average link quality changes observed on all links composing the available path $P_{k\left(\mathrm{n}_{0}, \mathrm{n}_{\mathrm{m}}\right)}$, with $m$ is the links number of the path $P_{k}$, given by:

$$
\beta=\sum_{n_{0}}^{n_{m}} \frac{\Delta P R R_{i, j}(t)}{m}
$$

It is directly proportional to the quality degradation: higher degradation means that the reception rate is decreased in some areas due to environment state changes, and so $\beta$ increases. $\beta$ decreases when the reception rate increases.

Let $\triangle P R R_{i, j}(\mathrm{t})=P R R_{i, j}(\mathrm{t})-P R R_{e n h_{i, j}}(\mathrm{t})$, where $P R R_{i, j}(\mathrm{t})$ is the standard PRR value, and $P R R_{-} e n h_{i, j}(\mathrm{t})$ is our proposed and enhanced PRR. 
From (5) and (6):

$$
\begin{aligned}
& \Delta P R R_{i, j}(\mathrm{t})=P R R_{i, j}(\mathrm{t})-P R R_{e n h_{i, j}}(\mathrm{t})=P R R_{i, j(t)}-\left(1-\left(\frac{e^{\frac{n_{-} \operatorname{loss}_{i, j}(t)}{\mathrm{max} \_\operatorname{loss}}}-1}{e-1}\right)\right) * P R R_{i, j}(\mathrm{t}) \\
& =\left(\frac{e^{\frac{n_{\text {_loss }}, j(t)}{\text { max }_{-} \text {loss }}}-1}{e-1}\right) * P R R_{i, j}(\mathrm{t})
\end{aligned}
$$

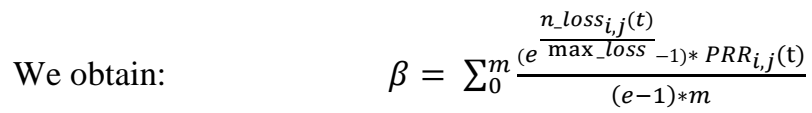

We rightly assume that: $\forall i, j \forall t / n_{-} \operatorname{loss}_{i, j}(t) \leq \max _{-}$loss. Then, $\quad \frac{e^{\frac{n_{-} \text {loss }_{i, j}(t)}{\text { max }_{-} l o s s}}-1}{e-1} \leq 1$

As we know that $\sum_{0}^{m} \frac{P R R_{i, j}(t)}{m} \leq 1$. We get $\beta \in[0,1]$

Finally, the links quality influences the stable path. Even if a path has good stability, when the path quality is low, the selection of the path with a good stability may be wrong because it may produce high data rate loss. We should ensure that $\frac{1}{2} \leq \beta<1$.

In order to ensure this condition, we obtain:

$$
\beta=\frac{1}{2}+\sum_{0}^{m} \frac{\Delta P R R_{i, j}(t)}{2 * m} \text {, where } m \text { is the number of links } l_{i, j} \text {. }
$$

The RREP message collects both $f q p_{k\left(\mathrm{n}_{0}, \mathrm{n}_{\mathrm{m}}\right)}$ and $f s p_{k\left(\mathrm{n}_{0}, \mathrm{n}_{\mathrm{m}}\right)}$ and updates their values at each intermediate node before reaching back toward the source node. Once RREP_Timer expires, the source node gets the value of $f_{k\left(n_{0}, n_{m}\right)}$ for all discovered paths. It sorts all paths according to the $f_{k\left(n_{0}, n_{m}\right)}$ value and selects the path having the maximum value, which represents the best path.

Furthermore, we may be in a situation where all the available paths have a poor quality; for instance, when the network has only a few or even just one available path. In this case, the network should avoid sending any data. For that purpose, we have defined a threshold $T h r$. If the $f_{k\left(n_{0}, n_{m}\right)}$ value of all available paths is below the threshold value, the source node will temporarily suspend data transmission for a specific time before checking again the available paths.

\subsection{Maintenance process}

In order to ensure node connectivity between any pair of nodes, the protocol uses HELLO packets, which are exchanged every $T_{\text {hello }}$ time. Due to the mobility of the nodes and environmental effects, a link failure may occur. In our model, a link is considered broken if the node does not receive $H$ successive HELLO messages from its neighbors. If a node has detected a route failure to a node $n_{i}$, it immediately deletes this link from its routing table, generates a RERR (Route Error) message containing $n_{i}$ as the unreached node, and sends the message back through the reverse path toward the source node. Every intermediate node receives the error message. Each node deletes the path to $n_{i}$ and any other paths that use $n_{i}$ as a next hop, adds the potential unreached nodes to the list of unreached nodes if any, and then forwards the RERR message toward the source node.

After the source node receives a RERR message, it removes the paths that correspond to the unreached nodes. The source node checks if it has an alternative path towards the destination; if no path is available, it starts a new discovery process. 

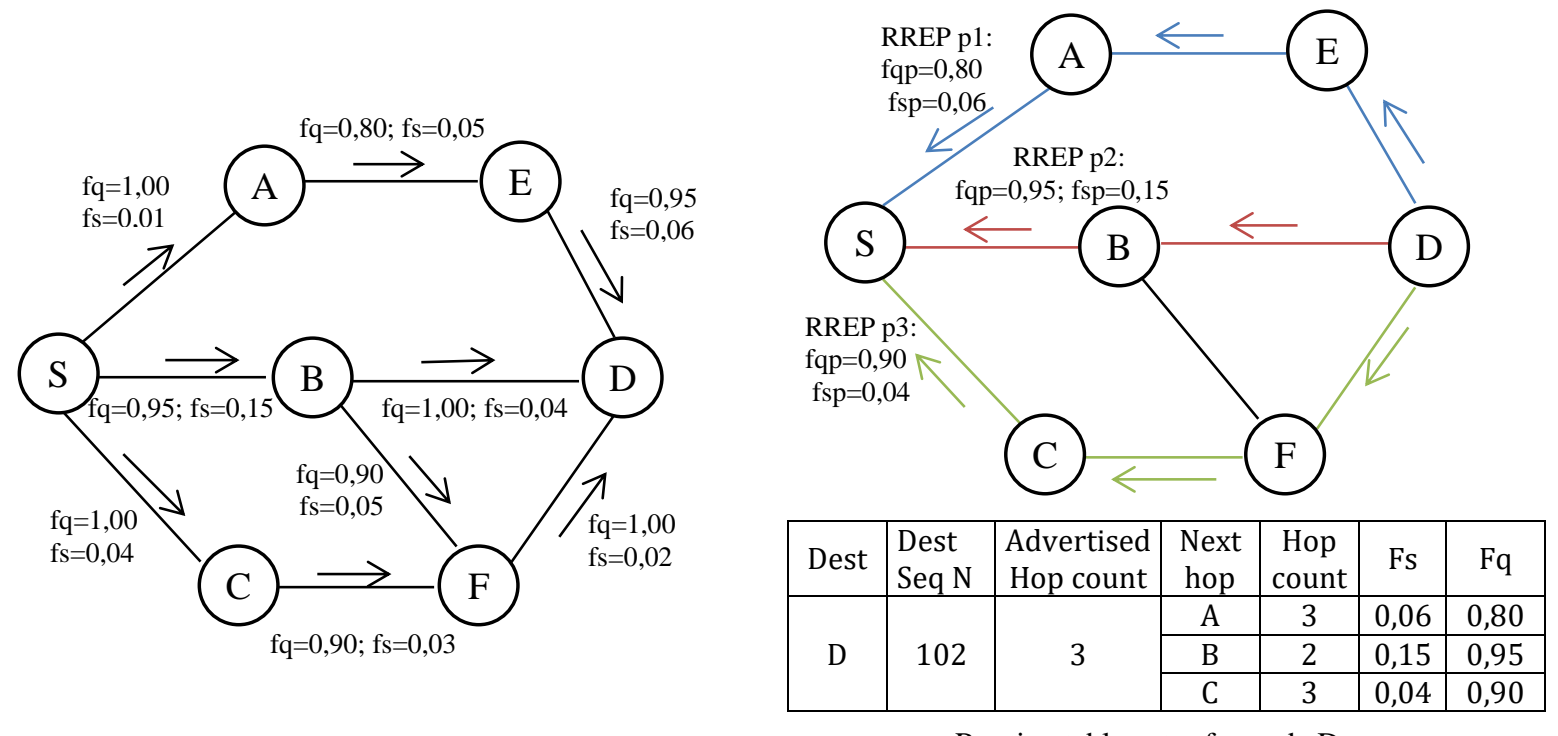

$\underline{\text { Routing table entry for node D }}$

Fig. 5. Example of our discovery and selection process

For example, in Fig. 5, when the timer expires for receiving RREP messages, our protocol calculates the objective function value of each discovered path (according to the algorithm 1 and 2) based on the routing table. The values of the objective function of the three paths are: 54.66, 59.66 and 64.00 respectively (according to the function 11). The source node $\mathrm{S}$ will select the third path to transmit data packets as it has the higher value. Finally, if a current path failure is detected (or the lack of its quality), the source node selects the next path for data transmission (second path in our example).

\subsection{Complexity analysis.}

The main drawbacks of a multipath routing protocol are that it may have a long convergence and a high complexity in the number of the exchanged messages. Due to the limited bandwidth of wireless links in an ad hoc network, the number of exchanged messages must be reduced. In addition, frequent changes of the ad hoc networks topology require that routing processes need to find paths quickly. We measure the performance of our solution mainly by its message complexity, expressed as functions of the nodes number $(\mathrm{N})$ in the traversed paths. The message complexity is the maximum number of messages used by our routing process in the worst case. For the discovered path of our ad hoc network with reactive routing, we assume that message complexity is $\mathrm{O}(\mathrm{N})$ size. The time complexity is the maximum number of steps required for the multipath discovery in the worst case. Indeed, we assume that the internal computation time is dominated by the message transmission time. The main processes of our approach are path discovery and their maintenance; these processes are based on the message broadcasting that takes $\mathrm{O}(\mathrm{N})$ time complexity. Finally, in our protocol, the destination node and the source node wait for a certain period of time before responding to discovery requests and launching path selection. These two waiting periods generate an additional time cost for our protocol, which is constant and independent of $\mathrm{N}$.

\section{Performance evaluation of RMQS-ua}

In this section, we present the evaluation and the simulation results of our proposed routing protocol. First, we describe the simulation environment, where we use two types of mobility models: RWP (Random Waypoint), Manhattan Grid, and SMOOTH mobility. Then we study the performance evaluation by comparing our protocol to some of the existing MANET routing protocols: AOMDVxDDR [22], DTRP [29], A-LSEA [28] (based on AOMDV instead of AODV), and ELE-AOMDV[36] (based on Electre Tri method integrated to AOMDV routing protocol). We include a discussion of the results. 


\subsection{Performance Metrics}

We evaluate the performance of the routing protocols according to the following metrics:

- Packet delivery ratio: it is the ratio of the number of data packets received by the destination and the number of data packets generated by the source node. It is calculated as following:

$$
\frac{N b r \text { of received data packet }}{\text { Nbr of sended data packet }} 100
$$

- Control packet overhead: it is the ratio of the total number of control packets sent by all the nodes composing the network and the number of data packets received, calculated as follow:

$$
\frac{N b r \text { routing packet transmitted }}{\text { Nbr of received data packet }}
$$

- Normalized energy consumption: it is the sum of the energy spent by all nodes during the simulation time over the ratio of received data packets.

$$
\frac{\sum(\text { Initial_energy }- \text { residual_energy })}{\text { Received data packet ratio }}
$$

- Throughput: known as the total of received data bits by time in Kilobits per second (Kbps), defined as follow:

$$
\frac{\sum(\mathrm{Nbr} \text { of received data packet } * \text { packet size } * 8)}{\text { time } * 1000}
$$

\subsection{Performance Environment}

In order to validate our protocol, we have implemented RMQS-ua using the NS2.35 [37] (Network Simulator 2.35). To do this, we integrate our solution into the AOMDV protocol, making the necessary changes as described in Section 3.3.

We have used Bonnmotion [38] tool which is a Java-based tool in order to generate different mobility scenarios.

Our objective is to make the simulation environment into a more realistic scenario, where the environment may affect the signal through external noise, interference, and node mobility. Due to the fact that NS2 does not model noise, we have implemented a noise module in the simulator that gives each link connecting two nodes a random noise value, which may change over time, with a probability of $P_{c}$ that may change the noise value each given $\Delta t$. This random change is due to unexpected electromagnetic phenomena. The SNR value calculated may lead to packet loss, and we define two thresholds, $S N R_{\min }$ and $S N R_{\max }$ as the following:

- If $\left(S N R<S N R_{\min }\right)$, the packet is definitively lost;

- $\quad$ Else if $\left(S N R \geq S N R_{\max }\right)$, the packet is definitively received;

- Else, the packet may be lost, with an error probability of $\frac{S N R_{\max }-S N R}{S N R_{\max }-S N R_{\min }}$.

The categorization of the various mobility models is based on their specific mobility characteristics, which can be classified into two classes: random models and non-random models [39]. The frequently used mobility model in MANETs simulations is the random models such as Random Waypoint model (RWP), in which nodes move independently to a randomly chosen destination with a randomly selected velocity. The simplicity of these models may have been one reason for its widespread use in simulations. However, MANETs may be used in different applications where complex mobility patterns exist. Hence, several researchers have focused on the alternative mobility models with different mobility characteristics where the movement of a node is more or less restricted by its history, or other nodes in the neighborhood or the environment such as Gauss-Markov Mobility Model, Column Mobility Model, and Manhattan Grid Model. 


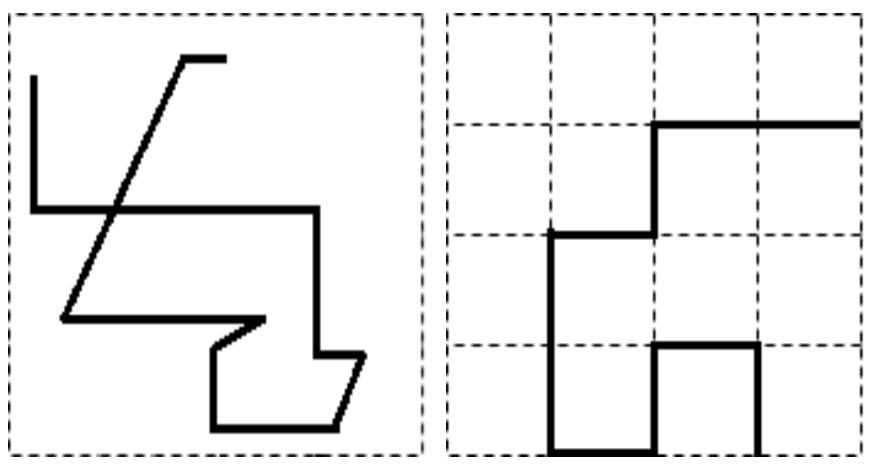

Fig. 6. Random Waypoint mobility model vs Manhattan Grid mobility model

Random Waypoint is the most widely used mobility model due to its simplicity. Initially, all mobile nodes are randomly placed in the area. Then, each node determines a random target location and starts moving toward it at a random speed. Once the node reaches that location, it waits for a specific time before repeating this process until the end of the simulation.

Manhattan Grid defines a mobility model characterized by a restricted moving area where the mobile nodes are allowed to move only on specific paths, which are determined by two parameters: the number of horizontal blocks $u$ and the number of vertical blocks $v$. The nodes have a pre-defined turn probability: once the node reaches an intersection, it may turn right or left with a probability $P_{\text {turn }}$ The Manhattan Grid model simulates the movement in an urban area containing streets.

As most past mobility models are not realistic, some researchers try to contribute in this context and propose a human mobility model. SMOOTH [40] is a recent mobility model that is realistic and based on several known features of human movement. The nodes do not move randomly but simulate real human walks. The concept is to create a specific number of sites named "clusters" (which represent communities) and get random popularity for each one. The mobile node was initially attributed randomly to clusters. A mobile node visits a few locations more frequently than other locations and may explore a new location with a specific probability or visits a previously visited location. During its movement, when a node reaches a location, it stays for a specific waited time before choosing another location.

For our contribution, we will use three mobility models: RWP (which describes free space mobility), Manhattan Grid (which describes city mobility), and SMOOTH (which describes human mobility behaviors). In order to make the Manhattan model more realistic in radio propagation, we have made some changes where two nodes separated by a block or more cannot communicate to each other even if they are in the transmission range.

\subsection{Simulation Results}

We study the performance of our routing protocol compared to the DTRP, A-LSEA, AOMDVxDDR, and ELEAOMDV protocols in order to determine the reliability and the accuracy of our solution. We have selected these reference protocols according to the following two criteria.

All comparison protocols are recent routing protocols for MANETs, which may provide good network performance. Additionally, the protocols have the same general characteristics: they are reactive, multipath, and based on link quality (AOMDVxDDR), link stability (A-LSEA), or multi constraints (DTRP and ELE-AOMDV).

We vary the number of network nodes from 50 to 200 to obtain different scenarios in an $1000 \mathrm{~m} \times 1000 \mathrm{~m}$ environment, ideal for ad hoc networks. The node speed varies from $5 \mathrm{~m} / \mathrm{s}$ to $20 \mathrm{~m} / \mathrm{s}$ (by increments of $5 \mathrm{~m} / \mathrm{s}$ in order to cover both low and high-speed mobility scenarios) with a pause time of $1 \mathrm{~s}$, these speeds reflect the reality of the nodes movements. We assume that the parameter values are $T h r=50 \%$ (as it represents a high probability to lose $50 \%$ of data packet; we deployed this parameter to eliminate the path having a low packet delivery rate (PDR)) and the number of successive packet losses indicating link failure (max_loss $=5$ ). For the data traffic, we use a CBR (Constant Bit Rate) with an inter-packet interval of $0.2 \mathrm{~s}$ (i.e., 5 packet/s). The radio model utilized is the Lucent WaveLAN [41]; each mobile node consumes $1000 \mathrm{~mW}$ at reception and $1400 \mathrm{~mW}$ at transmission. The initial energy of each node is 
randomly set between 30-100 J. For the noise module, we assume that $\Delta t=1.25 \mathrm{~s}$ (default value of AOMDV protocol, assigned to all simulated protocols) and the probability to change noise $P_{c}=0.03$; this probability value ensures a reasonable change of noise value in an urban area. We define $S N R_{\min }=5 \mathrm{~dB}$ and $S N R_{\max }=20 \mathrm{~dB}$ [42]. We use $\sigma=0.6$ in our simulation in order to favor recent distance measurements.

The values of the simulation parameters are summarized in Table 1.

\begin{tabular}{lll}
\hline Mobility model & Parameters & Value \\
\hline & Channel type & Wireless channel \\
& Routing protocols & RMQS-ua; DTRP; AOMDVxDDR; A-LSEA \\
& Simulation time & $400 \mathrm{~s}$ \\
& Number of mobile nodes & $50 / 100 / 200$ \\
& Radio propagation model & Two Ray Ground \\
& Network interface type & WirelessPhy \\
& MAC type & IEEE 802.11 \\
& Interface queue type & DropTail/PriQueue \\
& Max packet In Queue & 100 \\
& Terrain range & $1000 \mathrm{~m} \times 1000 \mathrm{~m}$ \\
& Transmission range & $250 \mathrm{~m}$ \\
\hline \multirow{2}{*}{ Random Waypoint } & RREP_Wait_Time & $0.5 \mathrm{~s}$ \\
& HELLO_Interval & $1.25 \mathrm{~s}$ \\
\hline \multirow{2}{*}{ Manhattan Grid } & Circular & False \\
& Nbrof Xblocks, Yblocks & 4,5 \\
& UpdateDist & $5.0 \mathrm{~s}$ \\
& TurnProb & 0.5 \\
& SpeedChangeProb & 0.2 \\
& MinSpeed and MaxSpeed & Varies from 5 to $20 \mathrm{~m} / \mathrm{s}$ \\
& PauseProb & 0.02 \\
& MaxPause & $1.0 \mathrm{~s}$ \\
\hline SMOOTH & Number of clusters & 10 \\
& $\left(\alpha, \mathrm{f}_{\text {min }}, \mathrm{f}_{\text {max }}\right.$ ) & $(2,50,500)$ \\
& $\left(\beta, p_{\text {min }}, p_{\text {max }}\right)$ & $(2,1,25)$ \\
\hline
\end{tabular}

Table 1 Simulation parameters

\subsubsection{Experiments based on the Manhattan Grid mobility model}

Fig. 7 presents the Packet Delivery Rate (PDR) with varying moving speeds for 50, 100, and 200 network nodes, based on the Manhattan Grid mobility model. The PDR reflects how the protocols react to the link connectivity and evaluate the data transmission reliability. The results indicate that RMQS-ua outperforms the compared protocols in all scenarios.

We notice that PDR for all protocols decreases with the increase of mobility speed, this makes nodes move out of transmission range faster leading to more link failures. RMQS-ua shows an interesting PDR for low number of network nodes with low speed by $10 \%$ over DTRP and AOMDVXDDR and 20\% over ELE-AOMDV; but with high speed scenario, RMQS-ua provides slight improvement by 5\%, 8\% compared to AOMDVxDDR and DTRP respectively because in this case, protocols tend to find only one path due to the restricted environment (based on Manhattan Grid model). Thus, there is no important impact on our protocol. ELE-AOMDV has less result and the worst PDR in 200 nodes scenario compared to others due to its path evaluation mechanism which do not include any stability or mobility metric. It uses the number of neighbors as a distance metric combined with energy and number of hops where the energy has the higher weight which represents a bad decision. A-LSEA has the worst PDR, especially for 50 nodes, due to a node elimination mechanism (based on link stability and energy on neighbor nodes during the discovery process) that may make a bad decision in a low-density network. Moreover, its stability function provides less accuracy in an urban environment due to its restricted mobility and signal reflection effect. 
(a) Number of network nodes: 50 nodes

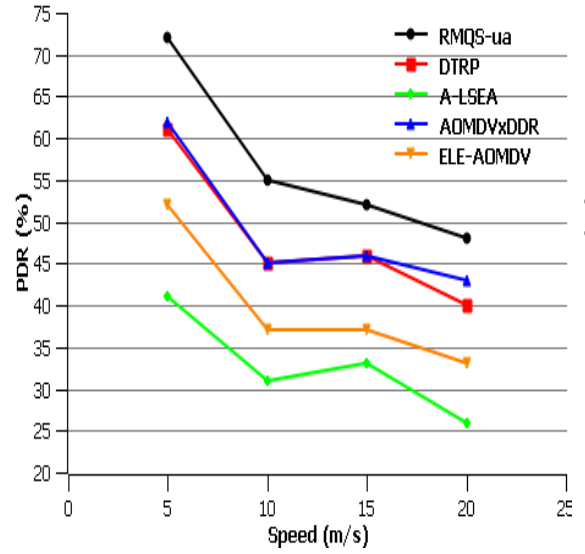

(b) Number of network nodes : 100 nodes

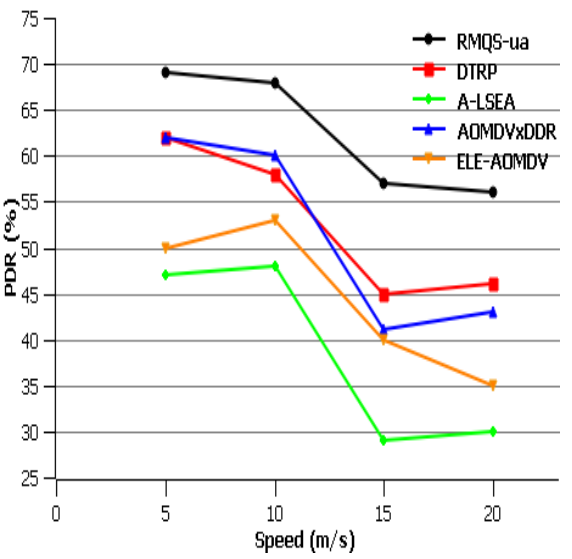

(c) Number of network nodes :200 nodes

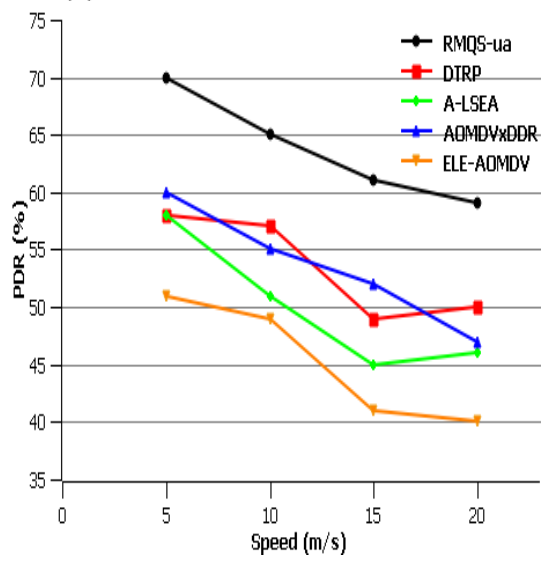

Fig. 7 Packet Delivery Rate vs node speed for Manhattan Grid

(a) Number of network nodes :50 nodes

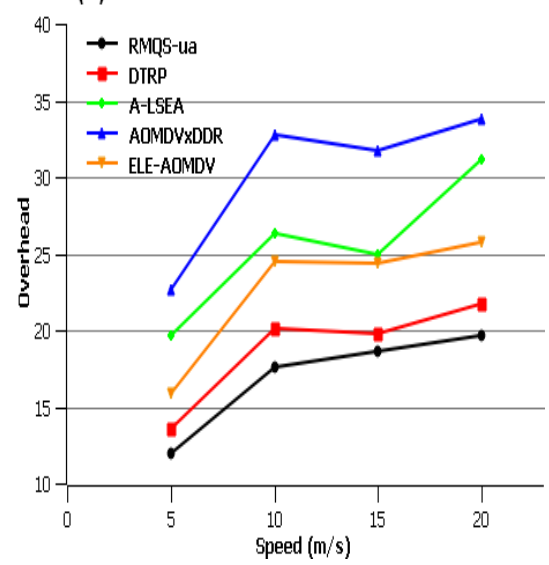

(b) Number of network nodes :100 nodes

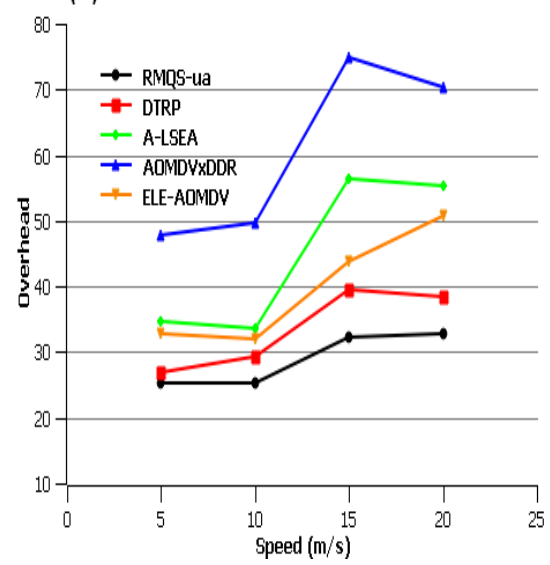

(c) Number of network nodes :200 nodes

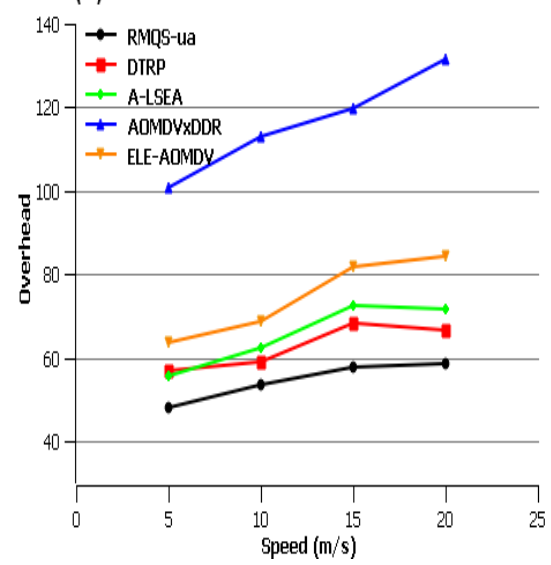

Fig. 8 Overhead rate vs node speed for Manhattan Grid

For 100 and 200 network nodes, we obtain a significant gain for successful data packet reception for RMQS-ua compared to other protocols, varying from $7 \%$ to $12 \%$ over DTRP, from $7 \%$ to $16 \%$ over AOMDVxDDR, from $7 \%$ to $25 \%$ over A-LSEA, and $15 \%$ to $20 \%$ over ELE-AOMDV.

These results show that our protocol ensures a reliable path selection, which increases the path lifetime by selecting the most stable path with satisfactory quality. Additionally, it detects and avoids the degraded links caused by unpredictable factors such as noise.

Another important performance metric is the control overhead, which is the number of control packets generated throughout the entire network during the simulation. The control overhead rate of all protocols is presented in Fig. 8 . We observe that RMQS-ua provides better results by reducing the overhead rate. The overhead rate of our proposition is $10 \%$ to $18 \%$ less than DTRP in most cases, $14 \%$ to $43 \%$ less than A-LSEA, $20 \%$ to $35 \%$ less than ELE-AOMDV, and up to $53 \%$ less than AOMDVxDDR. AOMDVxDDR protocol generates a high control overhead rate (average of 50\%) due to the pulse quality-based classification which generates extra control packets as pulse messages transmitted periodically by all nodes with a specific rate. It also uses unicast instead of broadcast, which considerably increases the overhead. Our protocol RMQS-ua significantly reduces route failures by selecting the most reliable path, which decreases the number of discovery processes caused by environmental effects, city street constraints, and node mobility. Thus, RMQS-ua improves network performance in a restricted environment. 
(a) Number of network nodes :50 nodes

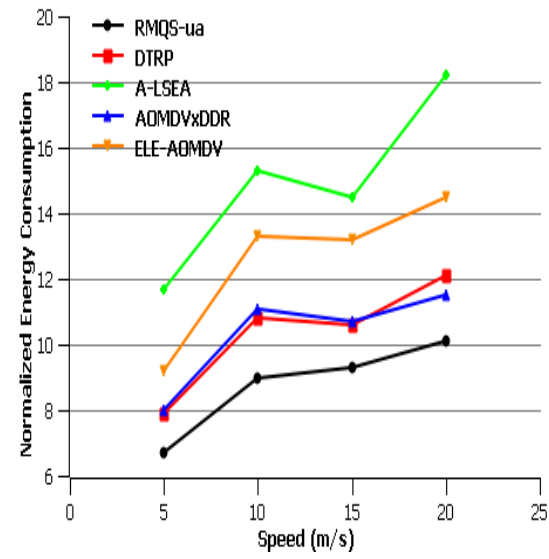

(b) Number of network nodes :100 nodes

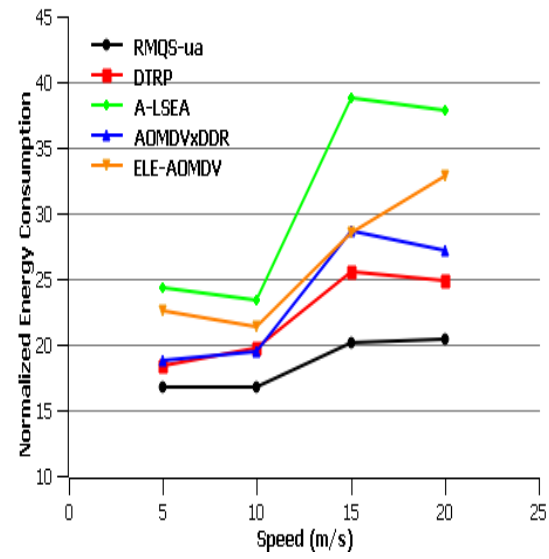

(c) Number of network nodes :200 nodes

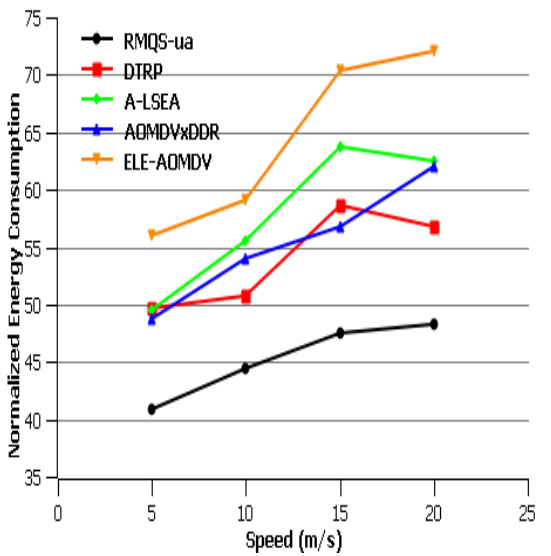

Fig. 9 Normalized energy consumption vs node speed for Manhattan Grid

(a) Number of network nodes :50 nodes

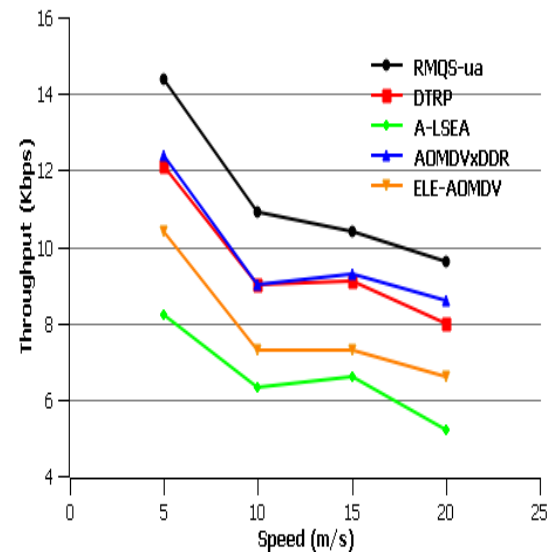

(b) Number of network nodes :100 nodes

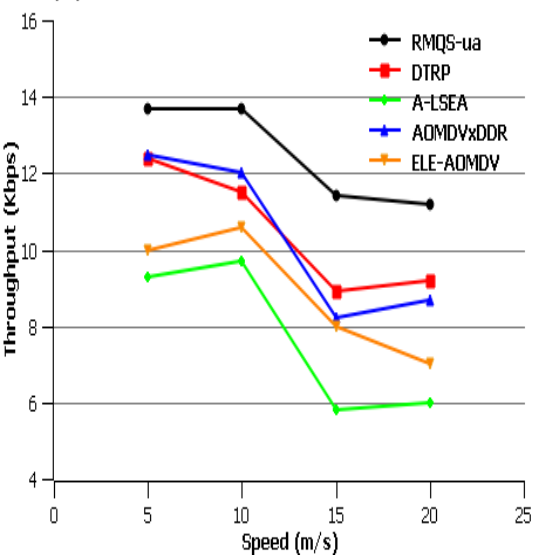

(c) Number of network nodes :200 nodes

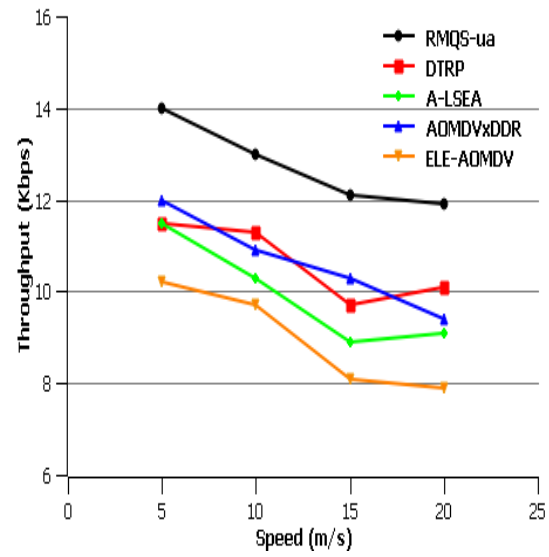

Fig. 10 Throughput vs node speed for Manhattan Grid

The normalized energy consumption is shown for various node speeds across different network sizes in Fig. 9. We can see that RMQS-ua conserves more energy compared to all other protocols. For 50 nodes (Fig. 9.a), RMQS-ua consumes less energy with normalized energy consumption improvement by $15 \%$ over DTRP and AOMDVxDDR. As the nodes number and the speed increase, the energy consumption increases; RMQS-ua provides better results compared to DTRP by $10 \%$ to $21 \%$, AOMDVxDDR by $10 \%$ to $30 \%$, ELE-AOMDV $21 \%$ to $37 \%$, and A-LSEA by $34 \%$ to $45 \%$ for 50 and 100 nodes, and by $18 \%$ to $23 \%$ for 200 nodes. The path selection principle of RMQS-ua protocol reduces the link failures and consequently reduces the control packet number and data packet loss which preserves energy consumption and prolongs the lifetime of individual nodes and hence the entire network lifetime.

Fig. 10 shows the comparison of our protocol RMQS-ua over the existing protocols in terms of throughput. The results show that the proposed protocol RMQS-ua has higher throughput compared to other protocols as it provides a better data packet delivery rate due to the path evaluation and selection mechanism based on link stability and link quality. We can note that RMQS-ua improves throughput by an average of $15 \%$ to $20 \%$ compared to DTRP and AOMDVxDDR, and more than 25\% compared to A-LSEA and ELE-AOMDV for most scenarios.

\subsubsection{Experiments based on the Random Waypoint mobility model}

Fig. 11 presents the simulation results of normalized energy consumption based on the Random Waypoint mobility model. RMQS-ua keeps its good performance in this mobility model too and consumes less energy compared to DTRP, AOMDVxDDR, A-LSEA, and ELE-AOMDV, with gains of $5 \%$ to $9 \%, 6 \%$ to $15 \%, 19 \%$ to $36 \%$, and $2 \%$ to $20 \%$ 
(a) Number of network nodes :50 nodes

(b) Number of network nodes :100 nodes

(c) Number of network nodes :200 nodes
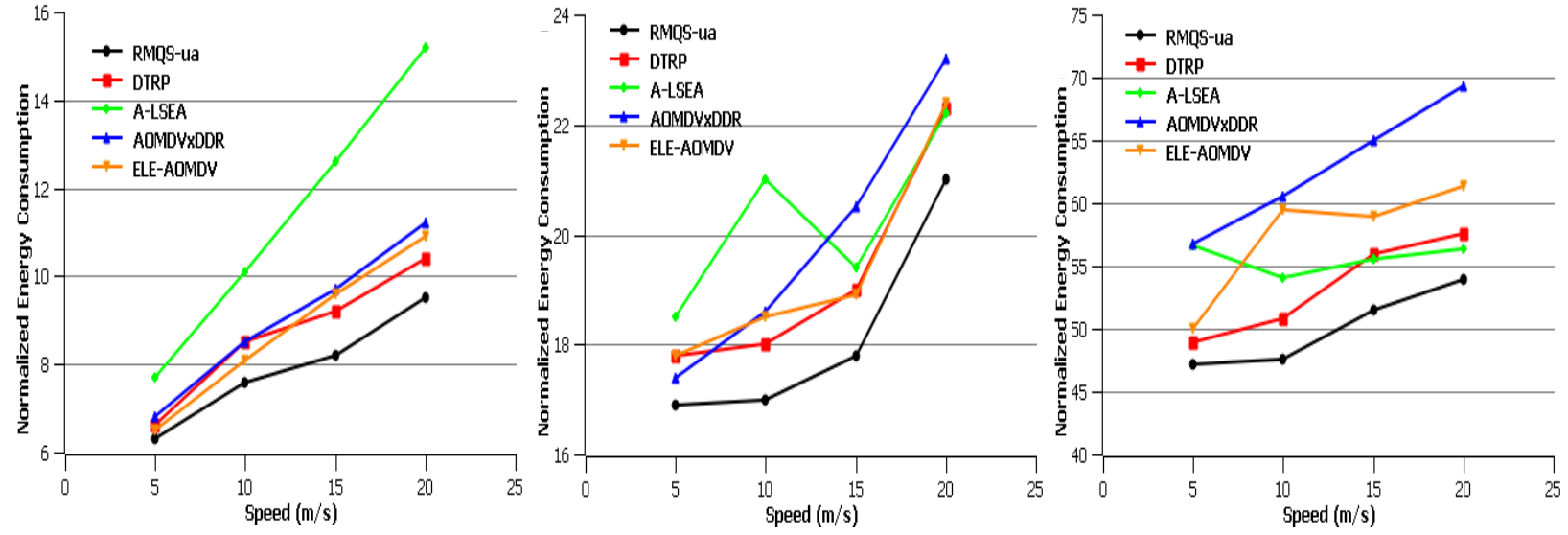

Fig. 11 Normalized energy consumption vs node speed for Random Waypoint

(a) Number of network nodes :50 nodes

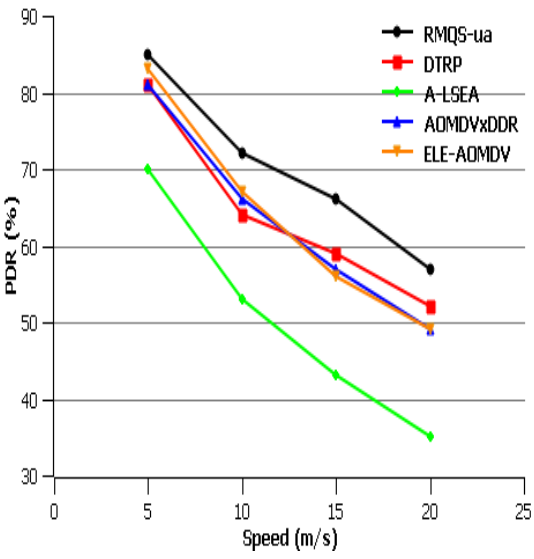

(b) Number of network nodes :100 nodes

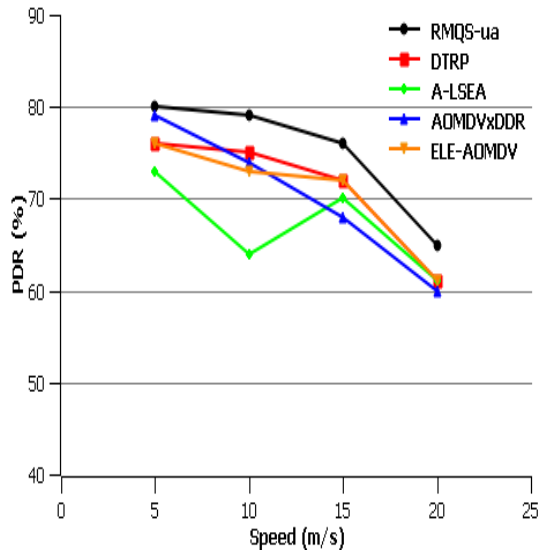

(c) Number of network nodes :200 nodes

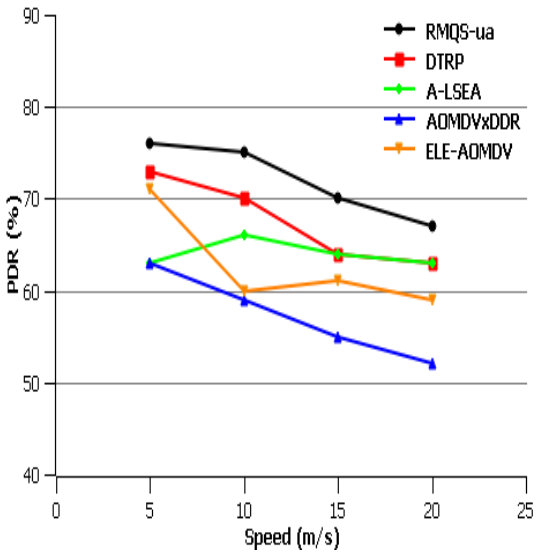

Fig. 12 Packet Delivery Rate vs node speed for Random Waypoint

respectively (as mentioned earlier, A-LSEA always gives the worst results for 50 and 100 nodes due to the elimination mechanism in low density network).

Fig. 12 shows that RMQS-ua presents a higher PDR than DTRP, AOMDVxDDR and ELE-AOMDV for low density network (Fig. 12.a) by a gain of $4 \%$ to $7 \%$ and a gain of $15 \%$ to $23 \%$ over A-LSEA for low node speed (A-LSEA still offers limited results). In 100 and 200 nodes scenarios, RMQS-ua provides an improvement of PDR about 4\% compared to DTRP and ELE-AOMDV, and range from $4 \%$ to $15 \%$ over A-LSEA and AOMDVxDDR. In the scenario with high speed mobility, A-LSEA gets better PDR than AOMDVxDDR as the fundamental cause of link failures (more frequently) is the nodes mobility which is considered by A-LSEA, but AOMDVxDDR is based on the link quality metric only. We notice that the A-LSEA stability mechanism adapts better to the Random Waypoint model contrary to the Manhattan Grid model.

The obtained results show that our protocol still offers better performance, which indicates that even in a free space environment, the RMQS-ua protocol always conserves its reliability.

\subsubsection{Experiments based on the SMOOTH mobility model}


(a) Packet delivery rate vs number of nodes

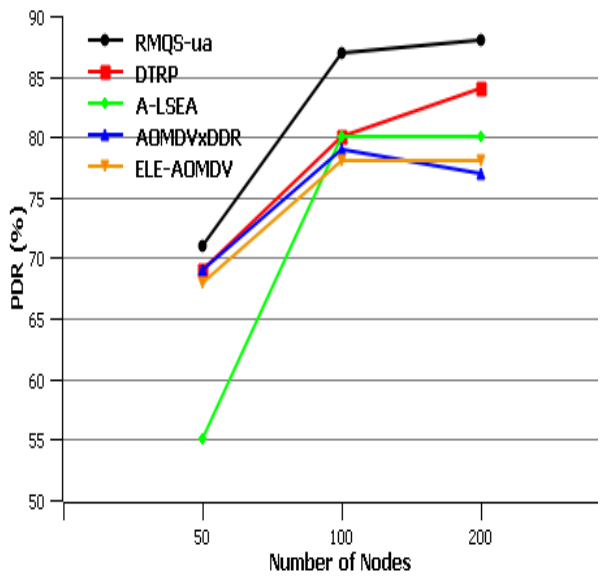

(c) Throughput vs number of nodes

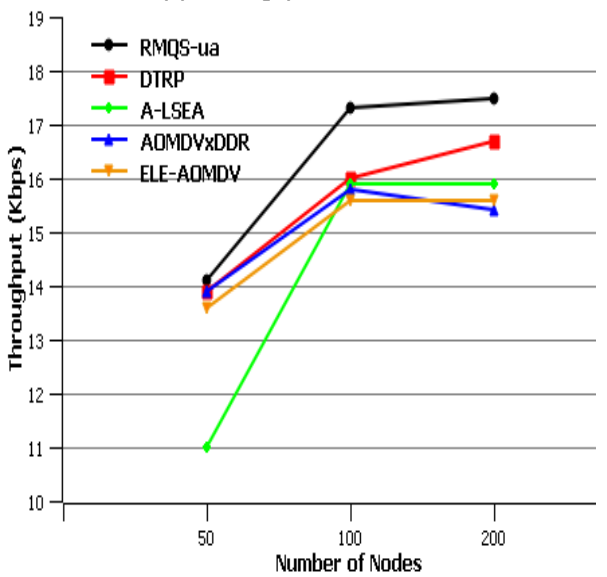

(b) Overhead vs number of nodes

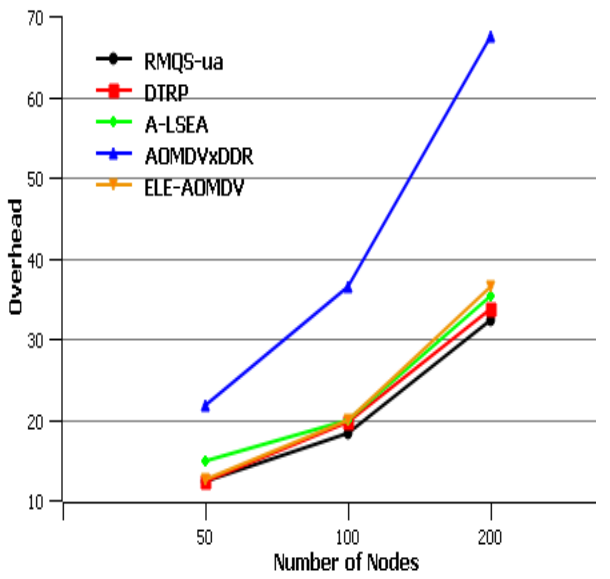

(d) Normalized Energy Consumption us number of nodes

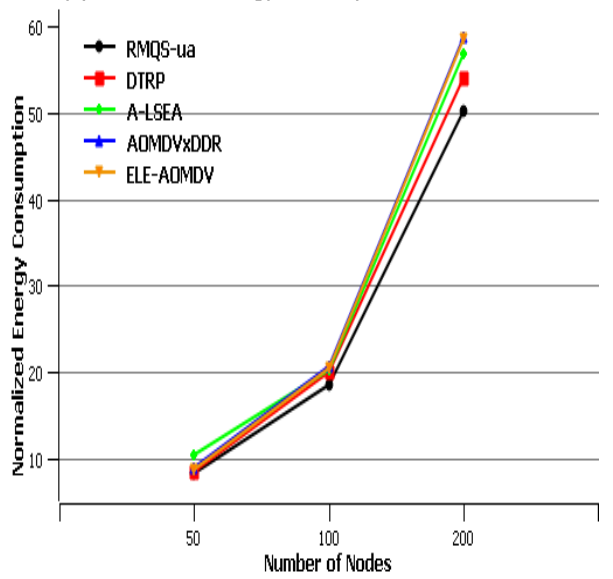

Fig. 13 Different performance metrics vs number of nodes for SMOOTH

Fig. 13 shows the different metrics of all protocols for 50,100 and 200 nodes in SMOOTH mobility model (representing human mobility). We can observe that RMQS-ua provides slight improvement for 50 nodes scenario for all performance metrics; generally, in this case, all protocols find only one or two paths between a source and a destination which makes the selection path process with less fact. In Fig. 13.a, RMQS-ua has a slight improvement of PDR by $2 \%$ compared to DTRP, AOMDVxDDR, and ELE-AOMDV for 50 nodes scenario. However, RMQS-ua provides better PDR as the number of nodes increase and outperforms other protocols by gain ranges from $7 \%$ to $10 \%$ in most cases (in 100 and 200 nodes). Fig. 13.b presents the overhead in different network densities; RMQS-ua reduces the overhead by 5\%, 8\%, $9 \%$ and 45\%compared to DTRP, A-LSEA, ELE-AOMDV, and AOMDVxDDR. RMQS-ua also reduces energy consumption by at least $8 \%$ compared to all other protocols as is shown in Fig. 13.d. This enhancement is due to the efficient evaluation path method used by our protocol and the selection of the optimal path to ensure reliable data transmission and reduce link failures.

Finally, our simulations show that our protocol, RMQS-ua, offers reliable data transmission and provides better performance in a variety of situations (high or low mobility, various node densities) independent of the mobility model (restricted city streets, free space environment, human behaviors).

\section{CONCLUSION}

A wireless network, especially an ad hoc network, may suffer from frequent link failures, which leads to a significant packet loss rate and increases the re-discovery process rate. This problem considerably affects the network performance, leading to higher energy consumption. 
To overcome this issue, and to ensure good network performance and improved reliability, we have proposed a new multipath routing protocol, RMQS-ua (Reliable Multipath Routing Protocol based on Link Quality and Stability in Urban areas). Our solution uses both link quality and link stability constraints to select the most efficient path for reliable data transmission. We have combined the SNR metric and a new, enhanced PRR metric for the quality metric measurement to allow us to detect link quality degradation and then avoid the worst paths.

We have simulated our protocol using two mobility models: Random Waypoint for open space, and Manhattan Grid, which provides realistic mobility behavior in an urban context. The evaluation shows the impact of our solution, which provides robustness and efficiency. We observe that the RMQS-ua protocol improves network performance and conserves more energy, which increases the network lifetime.

Next, we can try to explore other link quality and stability metrics to improve network performance. We are also trying to test our results on real-time data and check the result for validity. In the future, this work can be extended by proposing more efficient solutions in other fields such as QoS for complex urban environments containing hybrid networks with different wireless technologies.

In our future works, we will study the possibility to integrate other metrics in our approach such as energy and bandwidth. Moreover, our contribution is principally in the path evaluation and selection process. It will be interesting to extend our work to cover the path discovery process in order to preselect the nodes constituting the discovered paths. Finally, the evaluation of our protocol under real conditions is the main objective.

\section{REFERENCES}

[1] S. Naimi, A. Busson, V. Vèque, R. Bouallegue, Metric anticipation to manage mobility in mobile mesh and ad hoc wireless networks, Ann. Telecommun. 73 (2018) 787-802. https://doi.org/10.1007/s12243-018-0666-z.

[2] S.R. Malwe, N. Taneja, G.P. Biswas, Enhancement of DSR and AODV Protocols Using Link Availability Prediction, Wirel. Pers. Commun. 97 (2017) 4451-4466. https://doi.org/10.1007/s11277-017-4733-y.

[3] R. Prabha, N. Ramaraj, An improved multipath MANET routing using link estimation and swarm intelligence, EURASIP J. Wirel. Commun. Netw. 2015 (2015). https://doi.org/10.1186/s13638-015-0385-3.

[4] L. Hong, X. Liu, L. Zhang, W. Chen, Towards sensitive link quality prediction in ad hoc routing protocol based on grey theory, Wirel. Networks. 21 (2015) 2315-2325. https://doi.org/10.1007/s11276-015-0918-z.

[5] A.T. Tran, D.D. Mai, M.K. Kim, Link quality estimation in static wireless networks with high traffic load, J. Commun. Networks. 17 (2015) 370-383. https://doi.org/10.1109/JCN.2015.000068.

[6] A. Taha, R. Alsaqour, M. Uddin, M. Abdelhaq, T. Saba, Energy Efficient Multipath Routing Protocol for Mobile Ad-Hoc Network Using the Fitness Function, IEEE Access. 5 (2017) 10369-10381. https://doi.org/10.1109/ACCESS.2017.2707537.

[7] S.E. Benatia, O. Smail, B. Meftah, M. Rebbah, B. Cousin, An efficient energy aware link stable multipath routing protocol for mobile ad hoc networks in urban areas, Telfor J. 12 (2020) 2-7. https://doi.org/10.5937/telfor2001002e.

[8] N. Fareena, S. Sharmila Kumari, A distributed fuzzy multicast routing protocol (DFMCRP) for maximizing the network lifetime in mobile ad-hoc networks, J. Ambient Intell. Humaniz. Comput. (2020). https://doi.org/10.1007/s12652-020-01936w.

[9] C.V. Subbaiah, G. Kannayaram, Heuristic ant colony and reliable fuzzy QoS routing for mobile ad hoc network, J. Ambient Intell. Humaniz. Comput. (2021). https://doi.org/10.1007/s12652-021-03140-w.

[10] D. Palma, H. Araujo, M. Curado, Link quality estimation in wireless multi-hop networks using Kernel based methods, Comput. Networks. 56 (2012) 3629-3638. https://doi.org/10.1016/j.comnet.2012.07.012.

[11] A. Sharma, A. Bansal, V. Rishiwal, SBADR: stable and bandwidth aware dynamic routing protocol for mobile ad hoc network, Int. J. Pervasive Comput. Commun. 16 (2020) 205-221. https://doi.org/10.1108/IJPCC-05-2019-0043.

[12] L. Ghouti, Mobility prediction in mobile ad hoc networks using neural learning machines, Simul. Model. Pract. Theory. 66 (2016) 104-121. https://doi.org/10.1016/j.simpat.2016.03.001.

[13] M.V.S.S. Nagendranath, A.R. Babu, An efficient mobility aware stable and secure clustering protocol for mobile ADHOC networks, Peer-to-Peer Netw. Appl. 13 (2020) 1185-1192. https://doi.org/10.1007/s12083-019-00868-3.

[14] O. Smail, B. Cousin, R. Mekki, Z. Mekkakia, A multipath energy-conserving routing protocol for lifetime improvement of wireless ad hoc networks, Eurasip J. Wirel. Commun. Netw. (2014). https://doi.org/10.1186/1687-1499-2014-139.

[15] B. Nouha, K. Anis, M. Luca, Z. Marco Antonio, Y. Habib, B. Carlo Alberto, A. Mário, Radio Link Quality Estimation in Wireless Sensor Networks: a Survey, ACM Trans. Sen. Netw. 8 (2012) 1-33. https://doi.org/10.1145/2240116.2240123.

[16] A.M. Abdullah, E. Ozen, H. Bayramoglu, Investigating the impact of mobility models on MANET routing protocols, Int. J. 
Adv. Comput. Sci. Appl. 10 (2019) 25-35. https://doi.org/10.14569/ijacsa.2019.0100204.

[17] M. Appiah, The impact of mobility models on the performance of mobile ad hoc network (MANET), in: Int. Conf. Adv. Comput. Commun. Eng., IEEE, Durban, 2016: pp. 208-213. https://doi.org/10.1109/ICACCE.2016.8073749.

[18] M. Deb, S. Roy, B. Saha, P. Das, M. Das, Designing a new link quality estimator for sensor nodes by combining available estimators, in: 7th IEEE Int. Adv. Comput. Conf. IACC 2017, IEEE, Hyderabad, 2017: pp. 179-183. https://doi.org/10.1109/IACC.2017.0049.

[19] M.A. Moridi, M. Sharifzadeh, Y. Kawamura, H.D. Jang, Development of wireless sensor networks for underground communication and monitoring systems (the cases of underground mine environments), Tunn. Undergr. Sp. Technol. 73 (2018) 127-138. https://doi.org/10.1016/j.tust.2017.12.015.

[20] S. Jian, L. Song, L. Linlan, Z. Liqin, H. Gang, Research on link quality estimation mechanism for wireless sensor networks based on support vector machine, Chinese J. Electron. 26 (2017) 377-384. https://doi.org/10.1049/cje.2017.01.013.

[21] C.J. Lowrance, A.P. Lauf, Link Quality Estimation in Ad Hoc and Mesh Networks: A Survey and Future Directions, Wirel. Pers. Commun. 96 (2017) 475-508. https://doi.org/10.1007/s11277-017-4180-9.

[22] S.R. Afzal, S. Stuijk, M. Nabi, T. Basten, Effective link quality estimation as a means to improved end-to-end packet delivery in high traffic mobile ad hoc networks, Digit. Commun. Networks. 3 (2017) 150-163.

https://doi.org/10.1016/j.dcan.2016.12.001.

[23] G.M. De Araújo, A.R. Pinto, J. Kaiser, L.B. Becker, Genetic Machine Learning Approach for Link Quality Prediction in Mobile Wireless Sensor Networks, Coop. Robot. Sens. Networks. 507 (2014) 1-18. https://doi.org/10.1007/978-3-642-393013_1.

[24] Y.H. Robinson, S. Balaji, E.G. Julie, PSOBLAP: Particle Swarm Optimization - Based Bandwidth and Link Availability Prediction Algorithm for Multipath Routing in Mobile Ad Hoc Networks, Wirel. Pers. Commun. 106 (2019) 2261-2289. https://doi.org/10.1007/s11277-018-5941-9.

[25] A.S. Sharma, D.S. Kim, Energy efficient multipath ant colony based routing algorithm for mobile ad hoc networks, Ad Hoc Networks. 113 (2021). https://doi.org/10.1016/j.adhoc.2020.102396.

[26] A. Bhardwaj, H. El-Ocla, Multipath routing protocol using genetic algorithm in mobile ad hoc networks, IEEE Access. 8 (2020) 177534-177548. https://doi.org/10.1109/ACCESS.2020.3027043.

[27] M. Rajashanthi, K. Valarmathi, Energy-efficient multipath routing in networking aid of clustering with OGFSO algorithm, Soft Comput. 24 (2020) 12845-12854. https://doi.org/10.1007/s00500-020-04710-4.

[28] S. Hamad, S. Belhaj, M.M. Muslam, Average Link Stability with Energy-Aware routing protocol for MANETs, Int. J. Adv. Comput. Sci. Appl. 9 (2018) 554-562. https://doi.org/10.14569/IJACSA.2018.090176.

[29] O.S. Gnana Prakasi, P. Varalakshmi, Decision Tree Based Routing Protocol (DTRP) for Reliable Path in MANET, Wirel. Pers. Commun. 109 (2019) 257-270. https://doi.org/10.1007/s11277-019-06563-z.

[30] Z. Chen, W. Zhou, S. Wu, L. Cheng, An Adaptive on-Demand Multipath Routing Protocol with QoS Support for HighSpeed MANET, IEEE Access. 8 (2020) 44760-44773. https://doi.org/10.1109/ACCESS.2020.2978582.

[31] B.S. Kim, B.S. Roh, J.H. Ham, K. Il Kim, Extended OLSR and AODV based on multi-criteria decision making method, Telecommun. Syst. 73 (2020) 241-257. https://doi.org/10.1007/s11235-019-00609-0.

[32] G. Carofiglio, C.F. Chiasserini, M. Garetto, E. Leonardi, Route stability in MANETs under the random direction mobility model, IEEE Trans. Mob. Comput. 8 (2009) 1167-1179. https://doi.org/10.1109/TMC.2009.20.

[33] A. Moussaoui, F. Semchedine, A. Boukerram, A link-state QoS routing protocol based on link stability for Mobile Ad hoc Networks, J. Netw. Comput. Appl. 39 (2014) 117-125. https://doi.org/10.1016/j.jnca.2013.05.014.

[34] W. Sun, W. Lu, Q. Li, L. Chen, D. Mu, X. Yuan, WNN-LQE: Wavelet-Neural-Network-Based Link Quality Estimation for Smart Grid WSNs, IEEE Access. 5 (2017) 12788-12797. https://doi.org/10.1109/ACCESS.2017.2723360.

[35] M.K. Marina, S.R. Das, On-demand multipath distance vector routing in ad hoc networks, in: Proc. Ninth Int. Conf. Netw. Protoc. ICNP 2001, Riverside, CA, USA, 2001: pp. 14-23. https://doi.org/10.1109/ICNP.2001.992756.

[36] L. Aziz, S. Raghay, H. Aznaoui, Enhancing multipath routing using an efficient multicriteria sorting technique, Int. J. of Internet Technology and Secured Transactions 10 (2020) 675-693. https://doi.org/10.1504/JJITST.2020.110575

[37] The Network Simulator NS2, (n.d.). https://www.isi.edu/nsnam/ns/ (accessed March 20, 2020).

[38] BonnMotion - A mobility scenario generation and analysis tool, (n.d.). http://sys.cs.uos.de/bonnmotion/ (accessed November 19, 2020).

[39] F. Bai, A. Helmy, A Survey of Mobility Models in Wireless Adhoc Networks, Wirel. Ad Hoc Networks. (2004) 1-30.

[40] A. Munjal, T. Camp, W. C. Navidi, SMOOTH A Simple Way to Model Human Mobility, in Proceedings of the 14th ACM international conference on Modeling analysis and simulation of wireless and mobile systems MSWiM 2011. https://doi.org/10.1145/2068897.2068957.

[41] L.M. Feeney, M. Nilsson, Investigating the energy consumption of a wireless network interface in an ad hoc networking 
environment, in: IEEE INFOCOM Conf. Comput. Commun. Twent. Annu. Jt. Conf. IEEE Comput. Commun. Soc., Anchorage, AK, USA, 2001: pp. 1548-1557. https://doi.org/10.1109/infcom.2001.916651.

[42] M. Radi, B. Dezfouli, K.A. Bakar, S.A. Razak, M. Lee, Network initialization in low-power wireless networks: A comprehensive study, Comput. J. 57 (2014) 1238-1261. https://doi.org/10.1093/comjnl/bxt074. 